Article

\title{
Total Synthesis and Absolute Configuration of the Marine Norditerpenoid Xestenone
}

\section{Koichiro Ota, Takao Kurokawa, Etsuko Kawashima and Hiroaki Miyaoka *}

School of Pharmacy, Tokyo University of Pharmacy and Life Sciences, 1432-1 Horinouchi, Hachioji, Tokyo 192-0392, Japan; E-Mails: otak@toyaku.ac.jp (K.O.); kawasima@toyaku.ac.jp (E.K.)

* Author to whom correspondence should be addressed; E-Mail: miyaokah@toyaku.ac.jp; Tel.: +81-42-676-3080; Fax: +81-42-676-3073.

Received: 2 November 2009; in revised form: 19 November 2009 / Accepted: 23 November 2009 / Published: 24 November 2009

\begin{abstract}
Xestenone is a marine norditerpenoid found in the northeastern Pacific sponge Xestospongia vanilla. The relative configuration of C-3 and C-7 in xestenone was determined by NOESY spectral analysis. However the relative configuration of $\mathrm{C}-12$ and the absolute configuration of this compound were not determined. The authors have now achieved the total synthesis of xestenone using their developed one-pot synthesis of cyclopentane derivatives employing allyl phenyl sulfone and an epoxy iodide as a key step. The relative and absolute configurations of xestenone were thus successfully determined by this synthesis.
\end{abstract}

Keywords: xestenone; marine norditerpene; total synthesis; structural determination

\section{Introduction}

The norditerpenoid xestenone (Figure 1) was first isolated from the marine sponge Xestospongia vanilla in 1988 [1]. Its planar structure was determined by ${ }^{1} \mathrm{H}-$ and ${ }^{13} \mathrm{C}-\mathrm{NMR}$ and mass spectral analysis. The stereochemistry comprises two cis fused cyclopentane rings, as determined by the NOE correlation between the methyl protons at $\mathrm{C}-17$ and the methine proton at $\mathrm{C}-3$, although the relative configuration of C-12 and the absolute configuration were not determined. Moreover, no biological activity has been reported for xestenone, although various bioactive compounds that have been isolated from several Xestospongia sponges [2]. 
Figure 1. Structure of xestenone.

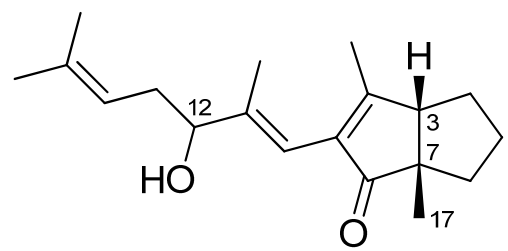

xestenone

The authors recently reported a stereocontrolled one-pot synthesis of cyclopentane derivatives possessing a quaternary carbon, which involved: 1) reaction of an anion derived from allyl phenyl sulfone with epoxy iodide I to give epoxysulfone II; 2) in situ deprotonation of II to generate an epoxysulfone anion III and 3) intramolecular cyclization to give cyclopentane derivative IV (Scheme 1) [3]. This one-pot synthesis of cyclopentane derivatives has now been applied to the total synthesis of xestenone, and in this paper the authors wish to report on the successful total synthesis of xestenone and its complete structural determination.

Scheme 1. One-pot synthesis of cyclopentane derivatives.<smiles>CC1(CCCI)OC1CCc1ccccc1</smiles>

I<smiles>C=C[C@]1(S(=O)(=O)c2ccccc2)CCC[C@@]1(C)[C@H](O)CCc1ccccc1</smiles>

IV

$\alpha$-sulfone : $\beta$-sulfone $=3.7: 1$

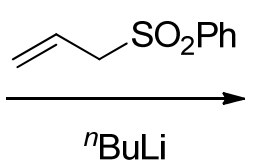

${ }^{n} \mathrm{BuLi}$

$84 \%$

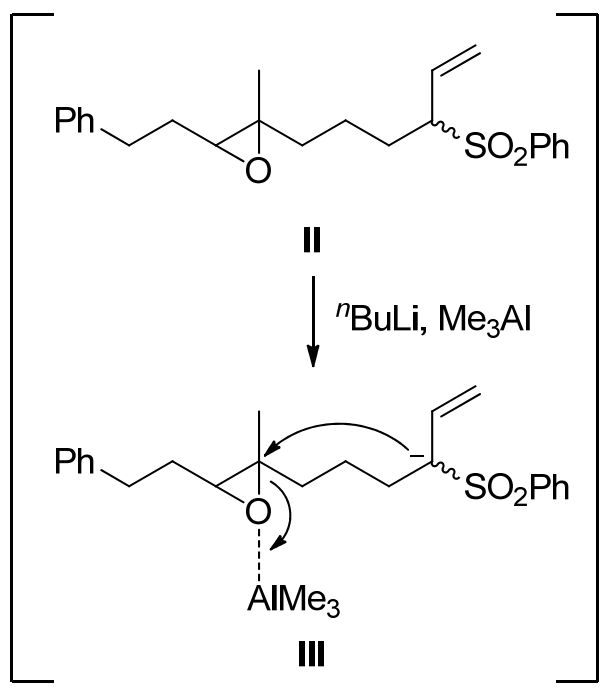

\section{Results and Discussion}

\subsection{Retrosynthetic analysis}

The authors planned to synthesize both xestenone and 12-epi-xestenone, since the relative configuration at $\mathrm{C}-12$ of xestenone was unknown at the onset (Scheme 2). Xestenone was obtained from secoxestenone by intramolecular aldol condensation [4]. Secoxestenone would be obtained from $\alpha, \beta$-unsaturated ketone $\mathbf{A}$ by 1,2-reduction of $\alpha, \beta$-unsaturated ketone at $\mathrm{C}$-12 and oxidation of the hydroxy group at $\mathrm{C}-2$ and $\mathrm{C}-8$. The $\alpha, \beta$-unsaturated ketone $\mathbf{A}$ would be obtained from aldehyde $\mathbf{B}$ by the Horner-Wadsworth-Emmons reaction using known phosphonate $\mathbf{C}$ [5]. For the right hand fragment of xestenone, aldehyde B would be synthesized from cyclopentane D through various chemical 
functionalizations. Cyclopentane D would be constructed by our developed stereocontrolled one-pot synthesis of cyclopentane derivatives using trisubstituted epoxy iodide $\mathbf{E}$ and allyl phenyl sulfone [3]. Epoxy iodide $\mathbf{E}$ would be obtained from alcohol $\mathbf{F}$.

Scheme 2. Retrosynthetic analysis of xestenone.

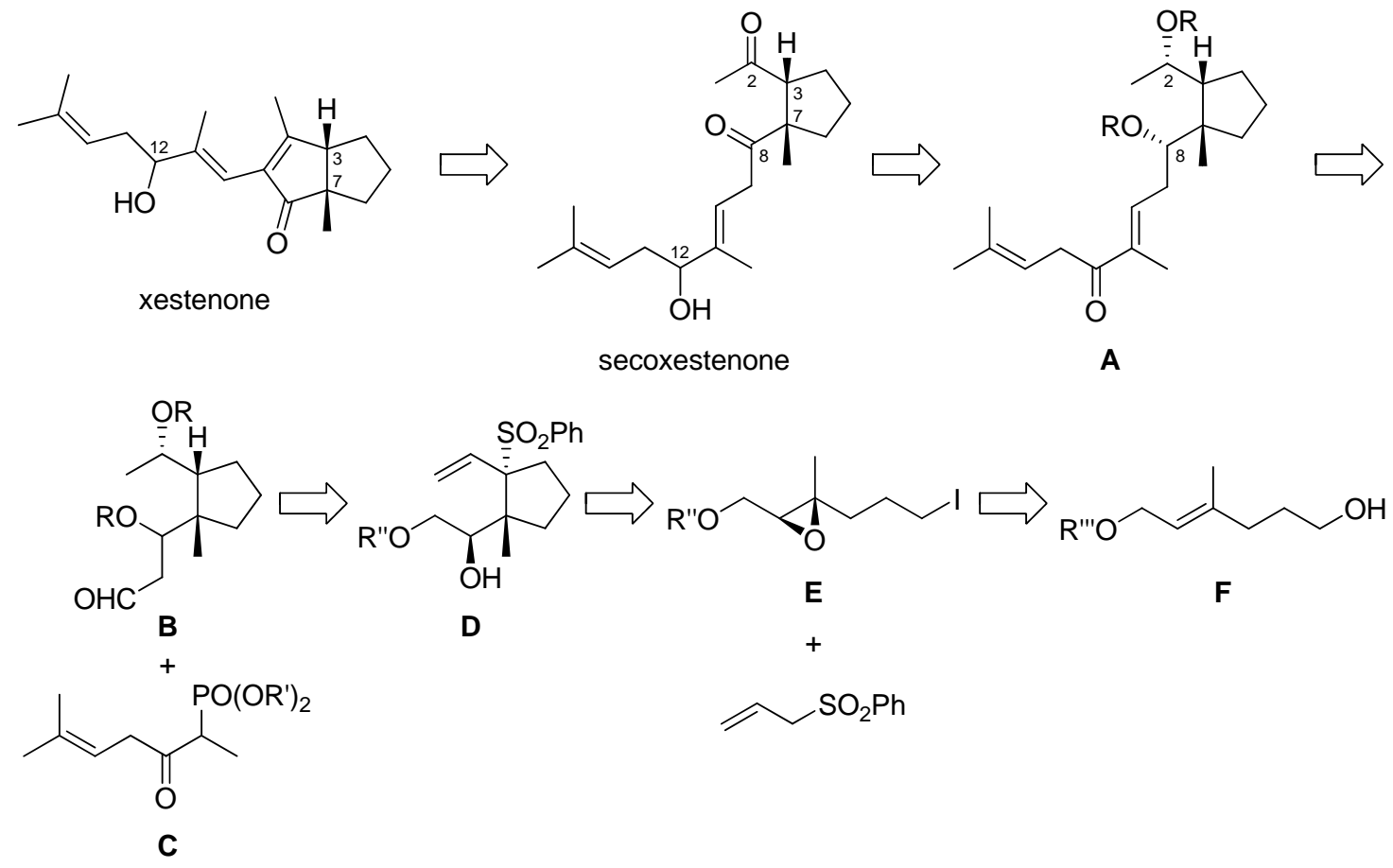

\subsection{Synthesis of xestenone}

Geraniol was converted to alcohol 1 by known procedures [6]. Alcohol 1 was treated with $p$-TsCl and pyridine to give the corresponding tosylate (90\%), which was deacetylated with $\mathrm{K}_{2} \mathrm{CO}_{3}$ in $\mathrm{MeOH}$ to furnish allylic alcohol 2 (94\%; Scheme 3). Allylic alcohol 2 was converted to chiral $\beta$-epoxyalcohol using Sharpless asymmetric epoxidation under standard conditions [7] (94\% ee). Iodination of the epoxy alcohol with $\mathrm{NaI}$ and $\mathrm{NaHCO}_{3}$ furnished epoxy iodide 3 (90\%, 2 steps). Protection of the primary hydroxy group in 3 as the TBS ether gave the desired chiral epoxy iodide 4 (95\%).

Scheme 3. Synthesis of epoxy iodide 4.

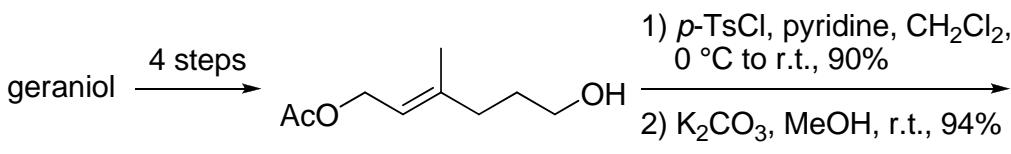<smiles>C/C(=C\CO)CCC[O+]</smiles>

2

1) TBHP, L-(+)-DIPT,

$\mathrm{Ti}\left(\mathrm{O}^{i} \mathrm{Pr}\right)_{4}, 4 \AA \mathrm{MS}$

$\mathrm{CH}_{2} \mathrm{Cl}_{2},-20^{\circ} \mathrm{C}$

2) $\mathrm{Nal}, \mathrm{NaHCO}_{3}$ acetone, r.t., $90 \%$ (94\%ee, 2 steps)<smiles>CC1(CCCI)OC1CO</smiles>

3

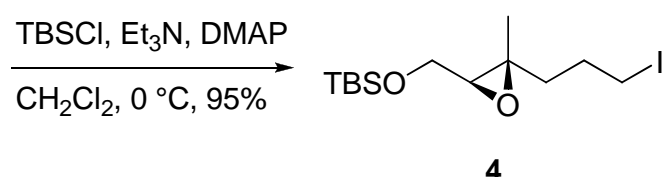


The sulfonyl carbanion prepared from allyl phenyl sulfone (2.3 eq.) and ${ }^{n} \mathrm{BuLi}$ (2.2 eq.) was reacted with epoxy iodide 4 at $-20{ }^{\circ} \mathrm{C}$. Following confirmation of the disappearance of 4 by TLC, ${ }^{n} \mathrm{BuLi}$ (1.2 eq.) and $\mathrm{Me}_{3} \mathrm{Al}$ (1.5 eq.) were added at $-78{ }^{\circ} \mathrm{C}$ to give cyclopentane 5 as the sole product (98\%; Scheme 4). The trans-configuration of the vinyl and 1-hydroxy-2-silyloxyethyl groups in cyclopentane 5 was determined by NOE correlation between the vinyl proton at C-2 and methyl protons at $\mathrm{C}-17$. The stereoselectivity of this reaction is presumably the result of steric hindrance between the phenyl sulfonyl group and the 1-hydroxy-2-silyloxyethyl group in the intermediate sulfonyl carbanion.

Scheme 4. Synthesis of intermediate 5.

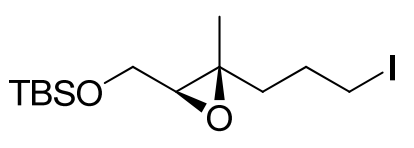

4

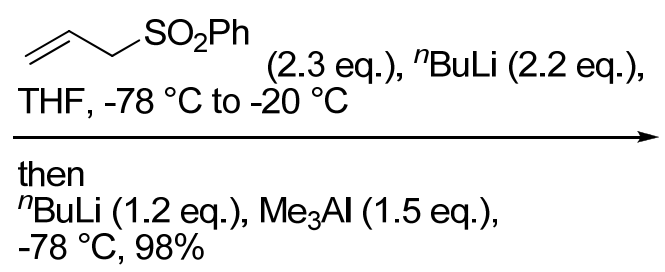

$-78{ }^{\circ} \mathrm{C}, 98 \%$

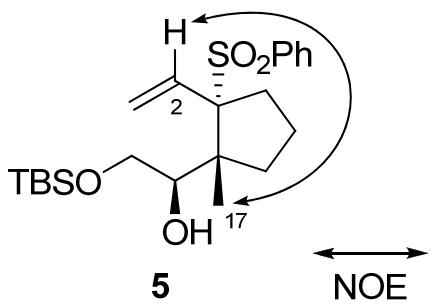

Protection of the secondary hydroxy group of 5 with TBSOTf and 2,6-lutidine (Scheme 5) furnished bis-silyl ether 6 (quant.). The phenylsulfonyl group in $\mathbf{6}$ was removed by treatment with $\mathrm{Na}(\mathrm{Hg})$ and $\mathrm{Na}_{2} \mathrm{HPO}_{4}$ to give trisubstituted $(E)$-olefin 7 as a sole product (quant.). The $E$-configuration of the trisubstituted olefin in 7 was determined by NOE correlation between the vinyl proton at C-2 and methyl protons at $\mathrm{C}-17$, and the vinyl proton at $\mathrm{C}-2$ and methylene protons at C-9. Diastereoselective hydroboration-oxidation of E-olefin 7 with catecholborane furnished a mixture of diol 8 and triol 8a. The stereochemistry of diol 8 was elucidated by NOESY spectral analysis. The NOE correlation between the methyl protons at $\mathrm{C}-17$ and methine proton at $\mathrm{C}-3$, and the methine proton at $\mathrm{C}-2$ and methine proton at $\mathrm{C}-8$ in diol 8 suggested that the methyl group and methine proton of cyclopentane were oriented in the same $\beta$-configuration. The mixture of diol $\mathbf{8}$ and triol $\mathbf{8 a}$ was treated with $p$ - $\mathrm{TsOH} \cdot \mathrm{H}_{2} \mathrm{O}$ in acetone to give acetonide 9 (95\%, 2 steps). Subsequent deprotection of the acetonide group in 9 and oxidative cleavage of the 1,2-diol with $\mathrm{HIO}_{4} \cdot 2 \mathrm{H}_{2} \mathrm{O}$ afforded hemiacetal 10, which was converted to homoallylic alcohols 11a (50\%, 2 steps) and 11b (36\%, 2 steps). These alcohols were easily separated by silica gel chromatography. The relative configurations of these homoallylic alcohols 11a and 11b were determined by chemical conversion and NOESY spectral analysis. Compounds 11a and 11b were converted to tetrahydrofurans 12a and 12b by treatment with $p$-TsCl, DMAP and $\mathrm{Et}_{3} \mathrm{~N}$ (Scheme 6). The NOE correlations of 12a between the methylene protons at C-9 and methyl protons at C-17, and one of the methylene protons at C-4 and methyl protons at C-1 suggested that the $\mathrm{C}-1$ methyl group and allyl group were oriented on different faces of the tetrahydrofuran ring, therefore, the stereochemistry of the hydroxy group at C-8 in homoallylic alcohol 11a was found to adopt a $\beta$-configuration. The NOE correlations of $\mathbf{1 2 b}$ between the methine proton at $\mathrm{C}-2$ and methine proton at $\mathrm{C}-3$, the methine proton at $\mathrm{C}-2$ and methine proton at $\mathrm{C}-8$, the methine proton at $\mathrm{C}-8$ and methyl protons at $\mathrm{C}-17$, the methine proton at $\mathrm{C}-3$ and methyl protons at $\mathrm{C}-17$, and the methyl protons at $\mathrm{C}-1$ and one of the methylene protons at $\mathrm{C}-4$ were observed. The results suggested that the C-1 methyl group and allyl group were oriented on the same face of the 
tetrahydrofuran ring. Therefore, the stereochemistry of the hydroxy group at C-8 in homoallylic alcohol 11b was found to adopt an $\alpha$-configuration. Both homoallylic alcohols 11a and 11b could be converted to xestenone. However, the chemical yield of the later steps in this synthesis from $\alpha$-alcohol 11b was low. Therefore, $\alpha$-alcohol 11b was converted into $\beta$-alcohol 11a by inversion of the C-8 stereocenter. The hydroxy group at $\mathrm{C}-2$ in $\alpha$-alcohol 11b was protected with TBDPSCl and imidazole to give TBDPS ether, which was oxidized with IBX [8] to afford the ketone. Diastereoselective reduction of the ketone with $\mathrm{NaBH}_{4}$ to the alcohol, followed by deprotection of the TBDPS group with TBAF furnished a mixture of the desired $\beta$-alcohol 11a (69\%, 4 steps) and $\alpha$-alcohol 11b (14\%, 4 steps).

Scheme 5. Synthesis of diols 11a and 11b.

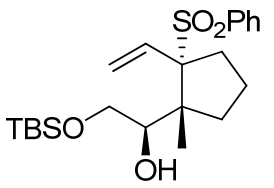

5

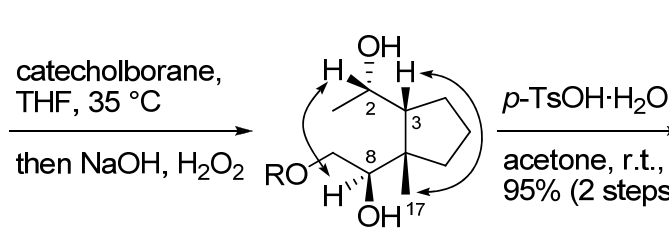

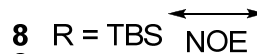

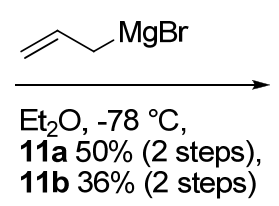

$8 \mathbf{a} \mathrm{R}=\mathrm{H}$
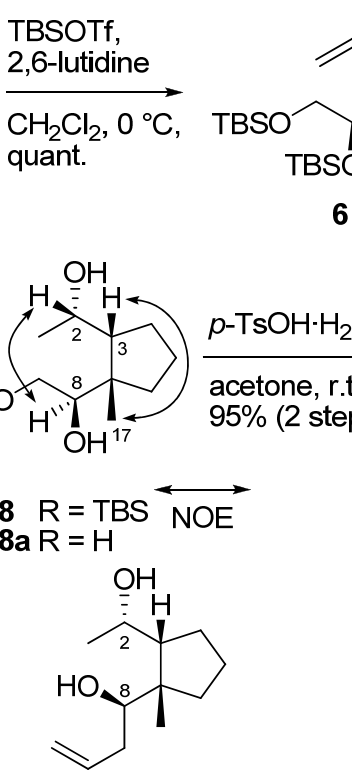

$11 a$

6
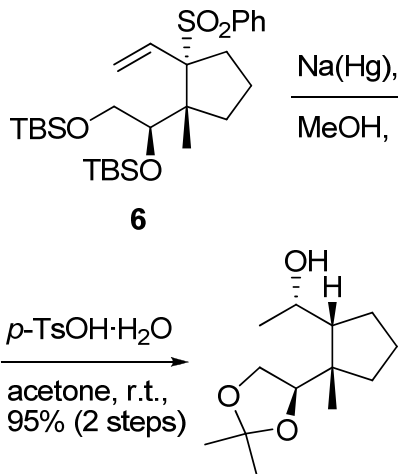

9<smiles>C=CC[C@@H](O)[C@]1(C)CCC[C@H]1[C@H](C)O</smiles>

$11 \mathrm{~b}$

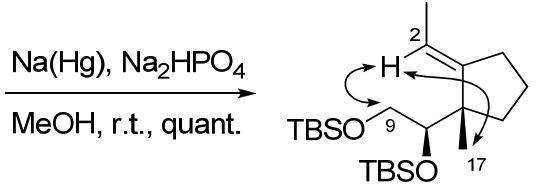

$7 \underset{\mathrm{NOE}}{\rightleftarrows}$

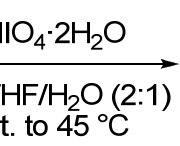

(1)

10

1) $T B D P S C l$, imid, DMF, r.t.

2) $\mathrm{IBX}, \mathrm{CH}_{3} \mathrm{CN}$, reflux

3) $\mathrm{NaBH}_{4}, \mathrm{MeOH}$, reflux

4) TBAF, THF, $40^{\circ} \mathrm{C}$,

11 a $69 \%$ (4 steps)

11b $14 \%$ (4 steps)

Scheme 6. Determination of the relative configuration of 11a and 11b.

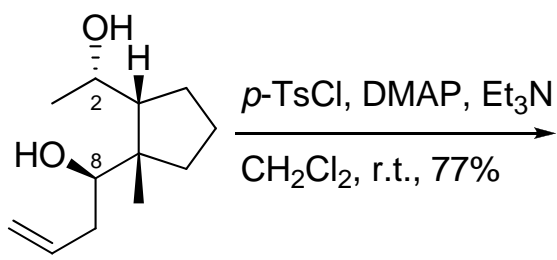

$11 \mathrm{a}$

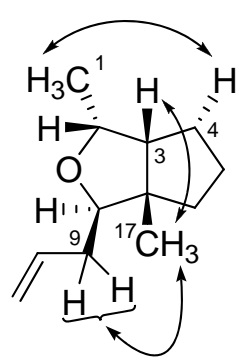

$12 a$

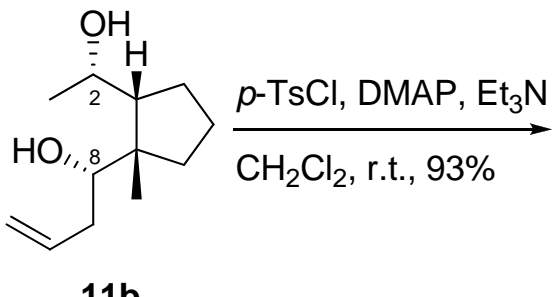

11b

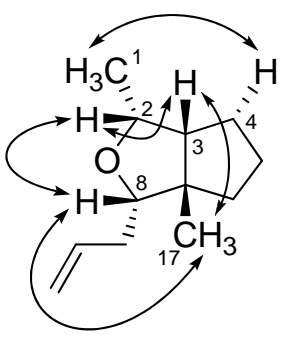

12b 
$\beta$-Alcohol 11a was converted to bis-silyl ether 13 by treatment with TBSOTf and 2,6-lutidine (99\%), and was followed by ozonolysis to give aldehyde 14 (quant.), thereby completing the synthesis of the right hand fragment of xestenone in 14 steps from known alcohol 1 [6] (Scheme 7). Treatment of aldehyde 14 with the anion of phosphonate 15 [5] in THF at r.t. provided $\alpha, \beta$-unsaturated ketone 16 (49\%). 1,2-Reduction of the $\alpha, \beta$-unsaturated ketone 16 with $\mathrm{NaBH}_{4}$ in the presence of $\mathrm{CeCl}_{3} \cdot 7 \mathrm{H}_{2} \mathrm{O}$ in $\mathrm{MeOH}$ [9] furnished allylic alcohol 17 as an inseparable mixture (99\%, 1:1). Protection of the hydroxy group in allylic alcohol 17 with $\mathrm{TrCl}$ and DMAP in pyridine provided the trityl ether, and was followed by desilylation with TBAF to give diol 18 (quant., 2 steps). Oxidation of two hydroxy groups in diol 18 with TFAA, DMSO and $\mathrm{Et}_{3} \mathrm{~N}$ generated diketone 19 (95\%). Lewis acid-mediated deprotection of the trityl group in diketone 19 with $\mathrm{Yb}(\mathrm{OTf})_{3}$ furnished diketone 20 as an inseparable diastereomeric mixture of the hydroxy group at C-12 (79\%). Diketone 20 corresponds to secoxestenone.

Scheme 7. Synthesis of diketone 20.

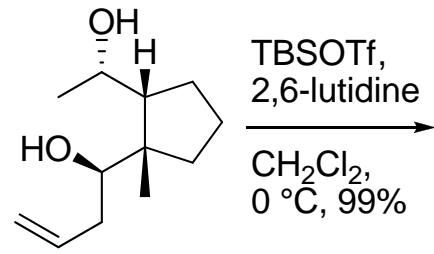

$11 a$

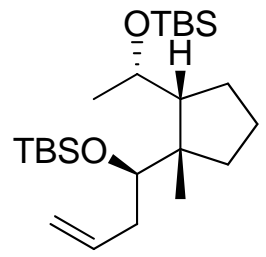

13

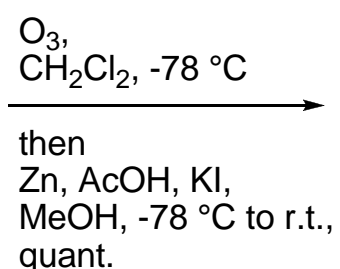
quant.<smiles>[CH][C@@]1([C@H](C)[OH2+])CCC[C@]1(C)[C@@H](CC=C(C)C(=O)CC=C(C)C)O[AsH3]</smiles>

16

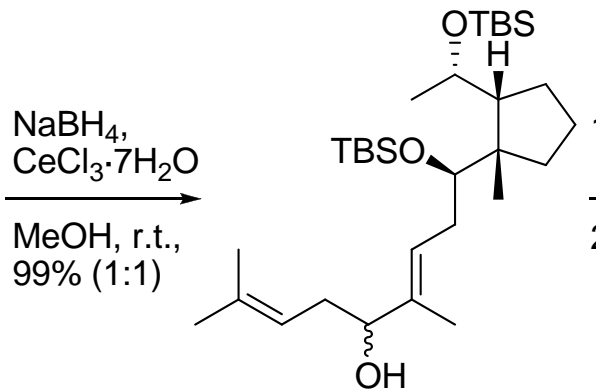

17

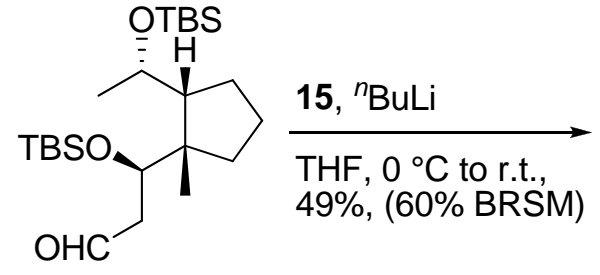

14

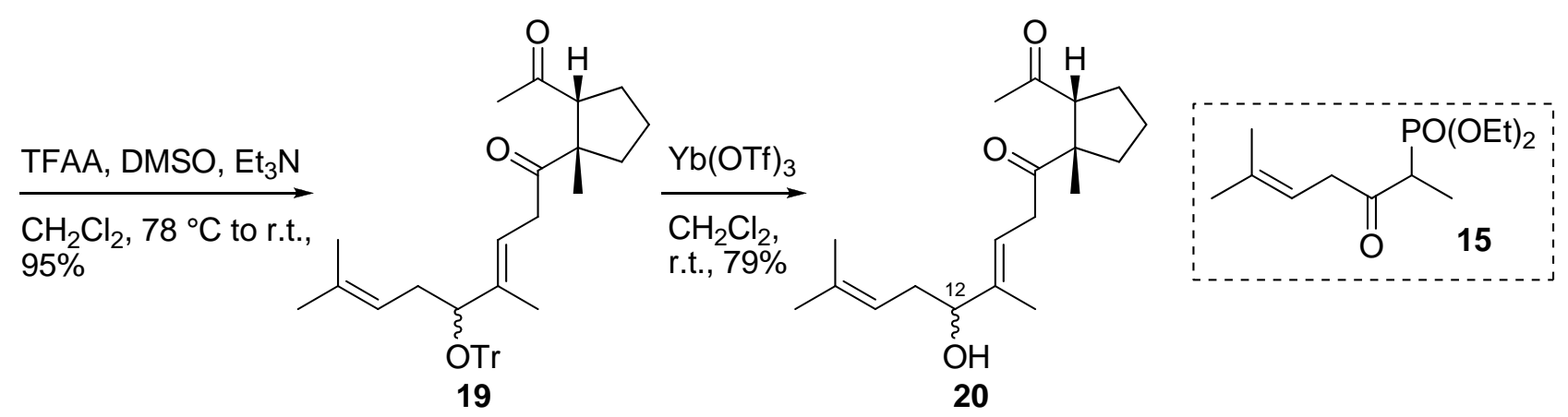

Finally, intramolecular aldol condensation of diketone 20 was achieved by treatment with $0.1 \mathrm{M}$ $\mathrm{NaOH}$ aq. to furnish a diastereomeric mixture of xestenone 21 and 12-epi-21 (88\%; Scheme 8). Separation of the diastereomeric mixture using a chiral HPLC column gave $21\left\{[\alpha]_{\mathrm{D}}{ }^{25}+2.2^{\circ}(c 0.08\right.$, $\mathrm{MeOH})\}$, and 12-epi-21 $\left\{[\alpha]_{\mathrm{D}}{ }^{25}-113.7^{\circ}(c 0.09, \mathrm{MeOH})\right\}$. The optical rotation of synthetic 21 is not 
identical, but very close to the value obtained for the natural product $\left\{[\alpha]_{\mathrm{D}} 0^{\circ}(c 1.00, \mathrm{MeOH})\right\}[1]$. Moreover, the $\mathrm{CD}$ spectrum of the synthetic 21 matched that of the natural product [1]. The CD spectrum of the synthetic 21 showed a positive Cotton effect at $323 \mathrm{~nm}$ and a negative Cotton effect at $258 \mathrm{~nm}$. The absolute configuration of the hydroxy group at C-12 in 21 was determined by comparing the ${ }^{1} \mathrm{H}-\mathrm{NMR}$ data of the two diastereomeric esters (MPA esters) [10]. The absolute configuration of the hydroxy group at C-12 in 12-epi-21 was determined by the same method. As a result, the absolute stereochemistry of the three chiral centers in xestenone was determined to be $3 S, 7 S$ and $12 R$. 12-epi-xestenone (12-epi-21) was converted to xestenone (21) by a Mitsunobu reaction.

Scheme 8. Synthesis of xestenone (21).

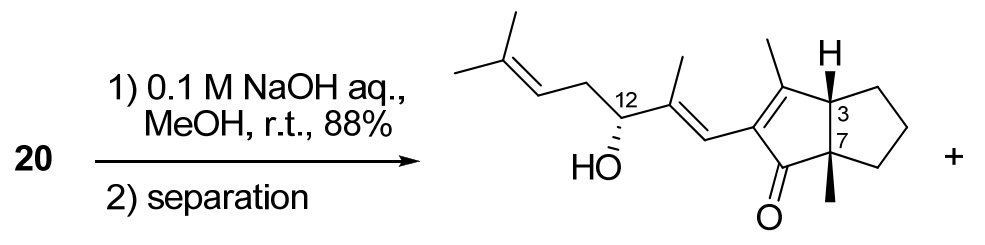

$(3 S, 7 S, 12 R)$

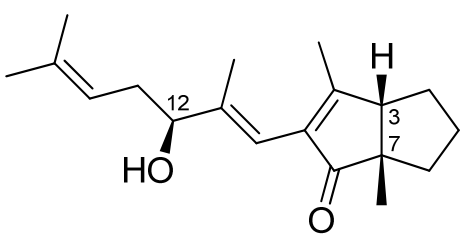

$(3 S, 7 S, 12 S)$

12-epi-xestenone (12-epi-21)

xestenone (21)

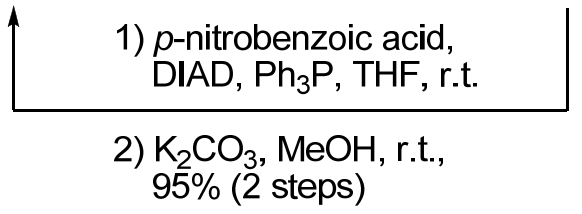

\section{Experimental Section}

\subsection{General}

Optical rotations were measured using a Jasco P-1030 polarimeter. Melting points (mp) were measured using a Yazawa melting point apparatus BY-2 and are uncorrected. IR spectra were recorded using a Jasco FT-IR/620 spectrometer. UV spectra were recorded using a Jasco V-550 spectrophotometer. Circular dichroism (CD) spectra were measured with a Jasco J-720 spectropolarimeter. ${ }^{1} \mathrm{H}$ - and ${ }^{13} \mathrm{C}-\mathrm{NMR}$ spectra were recorded on a Bruker DRX-400 or a Bruker Biospin AV-600 spectrometer. Chemical shifts are given on the $\delta(\mathrm{ppm})$ scale using tetramethylsilane (TMS) as the internal standard (s, singlet; d, doublet; t, triplet; q, quartet; quint, quintet; m, multiplet; br, broad). High resolution ESIMS (HRESIMS) spectra were obtained using a Micromass LCT spectrometer. Elemental analysis data were obtained using an Elemental Vavio EL. Flash column chromatography was performed using Kanto Chemical Silica Gel 60N (spherical, neutral) 40-50 $\mu \mathrm{m}$.

\section{2. (E)-6-Hydroxy-4-methylhex-4-enyl 4-methylbenzenesulfonate (2)}

To a solution of (E)-6-hydroxy-3-methylhex-2-enyl acetate [6] (1, $530 \mathrm{mg}, 3.08 \mathrm{mmol})$ in $\mathrm{CH}_{2} \mathrm{Cl}_{2}$ $(10.3 \mathrm{~mL})$ were added pyridine $(374 \mu \mathrm{L}, 4.62 \mathrm{mmol})$ and $p$-toluenesulfonyl chloride (705 $\mathrm{mg}$, 
$3.70 \mathrm{mmol}$ ) at $0{ }^{\circ} \mathrm{C}$. After stirring for $5 \mathrm{hr}$ at r.t., the mixture was diluted with $\mathrm{Et}_{2} \mathrm{O}$, washed with $\mathrm{H}_{2} \mathrm{O}$ and brine, and then dried. Removal of the solvent gave a residue which was then purified by silica gel column chromatography (hexane/AcOEt $=2: 1)$ to generate tosylate $(905 \mathrm{mg}, 90 \%$ yield) as a colorless oil. IR (neat) 2924, 1733, $1359 \mathrm{~cm}^{-1}$; ${ }^{1} \mathrm{H}-\mathrm{NMR}\left(400 \mathrm{MHz}, \mathrm{CDCl}_{3}\right) \delta \mathrm{ppm}: 7.77(2 \mathrm{H}, \mathrm{d}, J=8.2 \mathrm{~Hz}$ ), $7.33(2 \mathrm{H}, \mathrm{d}, J=8.2 \mathrm{~Hz}), 5.25(1 \mathrm{H}, \mathrm{m}), 4.52(2 \mathrm{H}, \mathrm{d}, J=7.2 \mathrm{~Hz}), 4.01(2 \mathrm{H}, \mathrm{t}, J=6.4 \mathrm{~Hz}), 2.44(3 \mathrm{H}, \mathrm{s})$, $2.05(2 \mathrm{H}, \mathrm{t}, J=7.2 \mathrm{~Hz}), 2.03(3 \mathrm{H}, \mathrm{s}), 1.77(2 \mathrm{H}, \mathrm{m}), 1.64(3 \mathrm{H}, \mathrm{s}) ;{ }^{13} \mathrm{C}-\mathrm{NMR}\left(100 \mathrm{MHz}, \mathrm{CDCl}_{3}\right) \delta \mathrm{ppm}$ : $170.9,144.7,133.3,129.8,127.8,119.6,69.8,61.0,35.0,26.8,21.5,20.9,16.2 ; \operatorname{HRESIMS}(\mathrm{m} / \mathrm{z})$ calcd. for $\mathrm{C}_{16} \mathrm{H}_{23} \mathrm{O}_{5} \mathrm{~S}(\mathrm{M}+\mathrm{H})^{+}$349.1086, found 349.1086; Anal. Calcd. for $\mathrm{C}_{16} \mathrm{H}_{22} \mathrm{O}_{5} \mathrm{~S}$ : C, 58.87; H, 6.79. Found: C, 58.94; H, 6.75.

To a solution of the above tosylate $(9.47 \mathrm{~g}, 29.0 \mathrm{mmol})$ in $\mathrm{MeOH}(290 \mathrm{~mL})$ was added $\mathrm{K}_{2} \mathrm{CO}_{3}$ (4.81 g, $34.8 \mathrm{mmol}$ ) at r.t. After stirring for $30 \mathrm{~min}$ at the same temperature, the mixture was diluted with $\mathrm{Et}_{2} \mathrm{O}$ and then filtered through a silica gel pad. Removal of the solvent gave a residue which was then purified by silica gel column chromatography (hexane/AcOEt $=2: 1$ ) to generate allylic alcohol 2 (7.74 g, 94\% yield) as a colorless oil. IR (neat) 3387, 2923, $1354 \mathrm{~cm}^{-1} ;{ }^{1} \mathrm{H}-\mathrm{NMR}\left(400 \mathrm{MHz}, \mathrm{CDCl}_{3}\right) \delta$ ppm: $7.75(2 \mathrm{H}, \mathrm{d}, J=8.2 \mathrm{~Hz}), 7.32(2 \mathrm{H}, \mathrm{d}, J=8.2 \mathrm{~Hz}), 5.31(1 \mathrm{H}, \mathrm{m}), 4.07(2 \mathrm{H}, \mathrm{d}, J=6.5 \mathrm{~Hz}), 4.00$ $(2 \mathrm{H}, \mathrm{t}, J=6.5 \mathrm{~Hz}), 2.42(3 \mathrm{H}, \mathrm{s}), 2.02(2 \mathrm{H}, \mathrm{t}, J=7.8 \mathrm{~Hz}), 1.76(2 \mathrm{H}, \mathrm{m}), 1.59(3 \mathrm{H}, \mathrm{s}), 1.52(1 \mathrm{H}, \mathrm{br} \mathrm{s})$; ${ }^{13} \mathrm{C}-\mathrm{NMR}\left(100 \mathrm{MHz}, \mathrm{CDCl}_{3}\right) \delta$ ppm: 144.7, 137.2, 133.2, 129.8, 127.8, 124.7, 69.8, 59.0, 35.0, 26.6, 21.5, 15.9; HRESIMS (m/z) calcd. for $\mathrm{C}_{14} \mathrm{H}_{21} \mathrm{O}_{4} \mathrm{~S}(\mathrm{M}+\mathrm{H})^{+}$307.0980, found 307.0994; Anal. Calcd. for $\mathrm{C}_{14} \mathrm{H}_{20} \mathrm{O}_{4} \mathrm{~S}$ : C, 59.13; H, 7.09. Found: C, 59.07; H, 7.06.

\section{3. ((2S,3S)-3-(3-Iodopropyl)-3-methyloxiran-2-yl)methanol (3)}

To a cold $\left(-20{ }^{\circ} \mathrm{C}\right)$ suspension of $4 \AA$ molecular sieves $(114 \mathrm{mg})$ in $\mathrm{CH}_{2} \mathrm{Cl}_{2}(1.6 \mathrm{~mL})$ were added L-(+)-DIPT $(5.2 \mu \mathrm{L}, 24.8 \mu \mathrm{mol}), \operatorname{Ti}\left(\mathrm{O}^{i} \operatorname{Pr}\right)_{4}(6.2 \mu \mathrm{L}, 21.0 \mu \mathrm{mol})$ and TBHP $(164 \mu \mathrm{L}, 101 \mathrm{mmol}, 6.17 \mathrm{M}$ in $\mathrm{CH}_{2} \mathrm{Cl}_{2}$ solution). After stirring for $30 \mathrm{~min}$ at the same temperature, a solution of allylic alcohol 2 (54.4 mg, $191 \mu \mathrm{mol})$ in $\mathrm{CH}_{2} \mathrm{Cl}_{2}(500 \mu \mathrm{L})$ was added over $5 \mathrm{~min}$. After stirring at $-20{ }^{\circ} \mathrm{C}$ for $15 \mathrm{~min}$, $\mathrm{NaOH}(13.0 \mu \mathrm{L}, 30 \%$ in saturated aqueous $\mathrm{NaCl})$ was added. The mixture was diluted with $\mathrm{Et}_{2} \mathrm{O}$, warmed to r.t. and stirred for $10 \mathrm{~min} . \mathrm{MgSO}_{4}(11.6 \mathrm{mg})$ and Celite $(1.4 \mathrm{mg})$ were then added and after stirring for $15 \mathrm{~min}$, the mixture was filtered through a Celite pad and the filtrate was concentrated under reduced pressure to afford the crude epoxide. To a solution of the crude epoxide in acetone $(1.9 \mathrm{~mL})$ were added $\mathrm{NaHCO}_{3}(17.7 \mathrm{mg}, 210 \mu \mathrm{mol})$ and $\mathrm{NaI}(286 \mathrm{mg}, 1.91 \mathrm{mmol})$ at r.t. After stirring for $8 \mathrm{hr}$ at the same temperature, the mixture was diluted with $\mathrm{Et}_{2} \mathrm{O}$ and then filtered through a silica gel pad. Removal of the solvent gave a residue which was then purified by silica gel column chromatography (hexane/AcOEt $=1: 2)$ to generate epoxy iodide $3(44.0 \mathrm{mg}, 90 \%$ yield) as a yellow oil. $[\alpha]_{\mathrm{D}}{ }^{28}-10.0\left(c\right.$ 1.03, $\mathrm{CHCl}_{3}$ ); IR (neat) 3418, $2929 \mathrm{~cm}^{-1} ;{ }^{1} \mathrm{H}-\mathrm{NMR}\left(400 \mathrm{MHz}, \mathrm{CDCl}_{3}\right) \delta \mathrm{ppm}$ : $3.79(1 \mathrm{H}, \mathrm{m}), 3.68(1 \mathrm{H}, \mathrm{m}), 3.18(2 \mathrm{H}, \mathrm{m}), 2.97(2 \mathrm{H}, \mathrm{t}, J=5.4 \mathrm{~Hz}), 1.99(1 \mathrm{H}, \mathrm{br} \mathrm{s}), 1.93(2 \mathrm{H}, \mathrm{m})$, $1.64(2 \mathrm{H}, \mathrm{m}), 1.29(3 \mathrm{H}, \mathrm{s}) ;{ }^{13} \mathrm{C}-\mathrm{NMR}\left(100 \mathrm{MHz}, \mathrm{CDCl}_{3}\right) \delta \mathrm{ppm}: 62.6,61.2,60.3,39.0,29.0,16.8,5.8$; HRESIMS $(\mathrm{m} / \mathrm{z})$ calcd. for $\mathrm{C}_{7} \mathrm{H}_{12} \mathrm{IO}(\mathrm{M}-\mathrm{OH})^{+}$238.9933, found 238.9930; Anal. Calcd. for $\mathrm{C}_{7} \mathrm{H}_{13} \mathrm{IO}_{2}$ : C, 32.83; H, 5.12. Found: C, 33.06; H, 5.26. 


\section{4. tert-Butyl(((2S,3S)-3-(3-iodopropyl)-3-methyloxiran-2-yl)methoxy)dimethylsilane (4)}

To a solution of epoxy iodide $3(387 \mathrm{mg}, 1.51 \mathrm{mmol})$ in $\mathrm{CH}_{2} \mathrm{Cl}_{2}(1.5 \mathrm{~mL})$ were added $\mathrm{Et}_{3} \mathrm{~N}$ (253 mg, $1.82 \mathrm{mmol}$ ), DMAP (185 mg, $1.51 \mathrm{mmol})$ and TBSCl (251 $\mathrm{mg}, 1.82 \mathrm{mmol})$ and the mixture was stirred at r.t. for $30 \mathrm{~min}$. The mixture was diluted with $\mathrm{Et}_{2} \mathrm{O}$, washed with saturated aqueous $\mathrm{NaHCO}_{3}$ solution, $\mathrm{H}_{2} \mathrm{O}$ and brine, and then dried. Removal of the solvent gave a residue which was then purified by silica gel column chromatography (hexane/AcOEt $=7: 1$ ) to generate epoxy iodide 4 (530 mg, 95\% yield) as a colorless oil. $[\alpha]_{\mathrm{D}}{ }^{25}+6.9$ (c 1.06, $\mathrm{CHCl}_{3}$ ); IR (neat) $2929 \mathrm{~cm}^{-1}$; ${ }^{1} \mathrm{H}-\mathrm{NMR}$ $\left(400 \mathrm{MHz}, \mathrm{CDCl}_{3}\right) \delta$ ppm: $3.76(1 \mathrm{H}, \mathrm{dd}, J=11.5,5.5 \mathrm{~Hz}), 3.69(1 \mathrm{H}, \mathrm{dd}, J=11.5,5.5 \mathrm{~Hz}), 3.19(2 \mathrm{H}, \mathrm{t}$, $J=7.0 \mathrm{~Hz}), 2.91(1 \mathrm{H}, \mathrm{t}, J=5.5 \mathrm{~Hz}), 1.95(2 \mathrm{H}, \mathrm{m}), 1.64(2 \mathrm{H}, \mathrm{m}), 1.26(3 \mathrm{H}, \mathrm{s}), 0.91(9 \mathrm{H}, \mathrm{s}), 0.07(6 \mathrm{H}$, s); ${ }^{13} \mathrm{C}-\mathrm{NMR}\left(100 \mathrm{MHz}, \mathrm{CDCl}_{3}\right) \delta \mathrm{ppm}: 62.8,62.0,59.5,39.0,29.2,25.9,18.3,16.7,5.8,-5.2,-5.4$; HRESIMS $(\mathrm{m} / \mathrm{z})$ calcd. for $\mathrm{C}_{13} \mathrm{H}_{28} \mathrm{IO}_{2} \mathrm{Si}(\mathrm{M}+\mathrm{H})^{+}$371.0903, found 371.0921; Anal. Calcd. for $\mathrm{C}_{13} \mathrm{H}_{27} \mathrm{IO}_{2} \mathrm{Si}$ : C, 42.16; H, 7.35. Found: C, 42.37; H, 7.23.

\section{5. (R)-1-[(1R,2S)-2-Benzenesulfonyl-1-methyl-2-vinylcyclopentyl]-2-(tert-butyldimethylsiloxy) ethanol (5)}

To a solution of allyl phenyl sulfone $(95.2 \mathrm{mg}, 0.552 \mathrm{mmol})$ in THF $(3.0 \mathrm{~mL})$ was added ${ }^{n} \mathrm{BuLi}$ (317 $\mu \mathrm{L}, 0.500 \mathrm{mmol}, 1.58 \mathrm{M}$ in hexane solution) at $-78{ }^{\circ} \mathrm{C}$ and the mixture was warmed to $0{ }^{\circ} \mathrm{C}$. The mixture was stirred for $30 \mathrm{~min}$ at the same temperature. After cooling to $-78{ }^{\circ} \mathrm{C}$, a solution of epoxy iodide $4(84.1 \mathrm{mg}, 0.227 \mathrm{mmol})$ in THF $(1.6 \mathrm{~mL})$ was added and the mixture was warmed to $-20{ }^{\circ} \mathrm{C}$. The mixture was stirred for $30 \mathrm{~min}$ at the same temperature. After cooling to $-78{ }^{\circ} \mathrm{C},{ }^{n} \mathrm{BuLi}$ $\left(173 \mu \mathrm{L}, 0.273 \mathrm{mmol}, 1.58 \mathrm{M}\right.$ in hexane solution) was added and the mixture was warmed to $-20{ }^{\circ} \mathrm{C}$. The mixture was stirred for $30 \mathrm{~min}$ at the same temperature. After cooling to $-78{ }^{\circ} \mathrm{C}, \mathrm{Me}_{3} \mathrm{Al}(331 \mu \mathrm{L}$, $0.341 \mathrm{mmol}, 1.03 \mathrm{M}$ in hexane) was added. After stirring for $1 \mathrm{hr}$ at the same temperature, the mixture was diluted with $\mathrm{Et}_{2} \mathrm{O}$, washed with saturated aqueous $\mathrm{NH}_{4} \mathrm{Cl}$ solution, $\mathrm{H}_{2} \mathrm{O}$ and brine, and then dried. Removal of the solvent gave a residue which was then purified by silica gel column chromatography (hexane/AcOEt $=10: 1)$ to generate cyclopentane $5(94.4 \mathrm{mg}, 98 \%$ yield) as a white solid. $\mathrm{mp}$ $125-126{ }^{\circ} \mathrm{C} ;[\alpha]_{\mathrm{D}}{ }^{25}-114\left(\right.$ c $\left.0.81, \mathrm{CHCl}_{3}\right)$; IR (KBr) 3560, $2952 \mathrm{~cm}^{-1} ;{ }^{1} \mathrm{H}-\mathrm{NMR}\left(400 \mathrm{MHz}, \mathrm{CDCl}_{3}\right) \delta$ ppm: $7.78(2 \mathrm{H}, \mathrm{m}), 7.57(1 \mathrm{H}, \mathrm{m}), 7.45(2 \mathrm{H}, \mathrm{m}), 6.19(1 \mathrm{H}, \mathrm{dd}, J=17.4,10.9 \mathrm{~Hz}), 5.25(1 \mathrm{H}, \mathrm{d}$, $J=10.9 \mathrm{~Hz}), 4.73(1 \mathrm{H}, \mathrm{d}, J=17.4 \mathrm{~Hz}), 4.56(1 \mathrm{H}, \mathrm{dt}, J=6.2,2.9 \mathrm{~Hz}), 4.29(1 \mathrm{H}, \mathrm{dd}, J=9.5,3.5 \mathrm{~Hz})$, $3.67(1 \mathrm{H}, \mathrm{t}, J=8.8 \mathrm{~Hz}), 3.23(1 \mathrm{H}, \mathrm{d}, J=2.9 \mathrm{~Hz}), 2.57(1 \mathrm{H}, \mathrm{m}), 2.06(3 \mathrm{H}, \mathrm{m}), 1.73(2 \mathrm{H}, \mathrm{m}), 0.99(3 \mathrm{H}$, s), $0.93(9 \mathrm{H}, \mathrm{s}), 0.14(6 \mathrm{H}, \mathrm{s}) ;{ }^{13} \mathrm{C}-\mathrm{NMR}\left(100 \mathrm{MHz}, \mathrm{CDCl}_{3}\right) \delta \mathrm{ppm}: 137.4,135.3,133.3,130.6,127.9$, 120.3, 81.0, 74.3, 64.2, 54.6, 37.2, 30.8, 25.9, 20.0, 19.6, 18.2, -5.1, -5.3; HRESIMS (m/z) calcd. for $\mathrm{C}_{22} \mathrm{H}_{37} \mathrm{O}_{4} \mathrm{SSi}(\mathrm{M}+\mathrm{H})^{+}$425.2182, found 425.2179; Anal. Calcd. for $\mathrm{C}_{22} \mathrm{H}_{36} \mathrm{O}_{4} \mathrm{SSi}: \mathrm{C}, 62.22 ; \mathrm{H}, 8.54$. Found: C, 62.11; H, 8.41.

\section{6. $\{(1 S, 2 R)-2-[(R)-1,2-B i s(t e r t-b u t y l d i m e t h y l s i l o x y) e t h y l]-2-m e t h y l-1-v i n y l c y c l o p e n t a n e s u l f o n y l\}$} benzene (6)

To a solution of cyclopentane $5(7.01 \mathrm{~g}, 16.5 \mathrm{mmol})$ in $\mathrm{CH}_{2} \mathrm{Cl}_{2}(16.5 \mathrm{~mL})$ were added 2,6-lutidine $(17.7 \mathrm{~g}, 165 \mathrm{mmol})$ and TBSOTf $(7.02 \mathrm{~g}, 26.6 \mathrm{mmol})$ and the mixture was stirred at $0{ }^{\circ} \mathrm{C}$ for $30 \mathrm{~min}$. 
The mixture was diluted with $\mathrm{Et}_{2} \mathrm{O}$, washed with saturated aqueous $\mathrm{NaHCO}_{3}$ solution, $\mathrm{H}_{2} \mathrm{O}$ and brine, and then dried. Removal of the solvent gave a residue which was then purified by silica gel column chromatography (hexane/AcOEt $=5: 1)$ to generate bis-silyl ether 6 (8.89 g, quantitative yield) as a colorless oil. $[\alpha]_{\mathrm{D}}{ }^{25}-77.9$ (c 1.62, $\mathrm{CHCl}_{3}$ ); IR (neat) 2954, $1133 \mathrm{~cm}^{-1} ;{ }^{1} \mathrm{H}-\mathrm{NMR}\left(400 \mathrm{MHz}, \mathrm{CDCl}_{3}\right) \delta$ ppm: $7.78(2 \mathrm{H}, \mathrm{m}), 7.57(1 \mathrm{H}, \mathrm{m}), 7.45(2 \mathrm{H}, \mathrm{m}), 6.37(1 \mathrm{H}, \mathrm{dd}, J=17.4,10.9 \mathrm{~Hz}), 5.27(1 \mathrm{H}, \mathrm{d}$, $J=10.9), 4.72(1 \mathrm{H}, \mathrm{d}, J=17.4 \mathrm{~Hz}), 4.63(1 \mathrm{H}, \mathrm{dd}, J=5.9,1.8 \mathrm{~Hz}), 4.15(1 \mathrm{H}, \mathrm{dd}, J=10.5,1.8 \mathrm{~Hz})$, $3.87(1 \mathrm{H}, \mathrm{dd}, J=10.5,5.9), 2.51(1 \mathrm{H}, \mathrm{m}), 2.12(1 \mathrm{H}, \mathrm{m}), 2.01(1 \mathrm{H}, \mathrm{m}), 1.90(3 \mathrm{H}, \mathrm{m}), 0.92(21 \mathrm{H}, \mathrm{m})$, $0.16(12 \mathrm{H}, \mathrm{m}) ;{ }^{13} \mathrm{C}-\mathrm{NMR}\left(100 \mathrm{MHz}, \mathrm{CDCl}_{3}\right) \delta \mathrm{ppm}: 137.3,135.3,133.2,130.7,127.9,120.7,81.1$, 77.2, 66.5, 56.3, 40.0, 31.6, 26.3, 26.2, 19.8, 19.0, 18.5, -3.3, -4.6, -5.1, -5.4; HRESIMS (m/z) calcd. for $\mathrm{C}_{28} \mathrm{H}_{51} \mathrm{O}_{4} \mathrm{SSi}_{2}(\mathrm{M}+\mathrm{H})^{+}$539.3047, found 539.3086; Anal. Calcd. for $\mathrm{C}_{28} \mathrm{H}_{50} \mathrm{O}_{4} \mathrm{SSi}_{2}$ : C, 62.40; $\mathrm{H}$, 9.35. Found: $\mathrm{C}, 62.37 ; \mathrm{H}, 9.11$.

\section{7. (S)-1-[(R)-1,2-Bis(tert-butyldimethylsiloxy)ethyl]-2-ethylidene-1-methylcyclopentane (7)}

To a solution of bis-silyl ether $6(9.29 \mathrm{~g}, 17.2 \mathrm{mmol})$ in $\mathrm{MeOH}(344 \mathrm{~mL})$ were added $\mathrm{Na}_{2} \mathrm{HPO}_{4}$ (17.1 g, $120.5 \mathrm{mmol})$ and $5 \% \mathrm{Na}(\mathrm{Hg})(31.6 \mathrm{~g})$. After stirring for $1 \mathrm{hr}$ at r.t., the mixture was diluted with $\mathrm{Et}_{2} \mathrm{O}$ and filtered through silica gel. The filtrate was then concentrated under reduced pressure. The resultant residue was then purified by silica gel column chromatography (hexane only) to generate E-olefin 7 (6.86 g, quantitative yield) as a colorless oil. $[\alpha]_{\mathrm{D}}{ }^{25}+19.0$ (c 1.35, $\mathrm{CHCl}_{3}$ ); IR (neat) $2955 \mathrm{~cm}^{-1}$; ${ }^{1} \mathrm{H}-\mathrm{NMR}\left(400 \mathrm{MHz}, \mathrm{CDCl}_{3}\right) \delta \mathrm{ppm}: 5.17(1 \mathrm{H}, \mathrm{m}), 3.78(1 \mathrm{H}, \mathrm{dd}, J=10.3,2.8 \mathrm{~Hz}), 3.52$ $(1 \mathrm{H}, \mathrm{dd}, J=9.2,1.8 \mathrm{~Hz}), 3.46(1 \mathrm{H}, \mathrm{dd}, J=10.3,5.8 \mathrm{~Hz}), 2.35(1 \mathrm{H}, \mathrm{m}), 2.08(2 \mathrm{H}, \mathrm{m}), 1.67(1 \mathrm{H}, \mathrm{m})$, $1.58(3 \mathrm{H}, \mathrm{d}, J=6.7 \mathrm{~Hz}), 1.53(2 \mathrm{H}, \mathrm{m}), 1.22(1 \mathrm{H}, \mathrm{m}), 0.97(3 \mathrm{H}, \mathrm{s}), 0.88(9 \mathrm{H}, \mathrm{s}), 0.86(9 \mathrm{H}, \mathrm{s}), 0.07(3 \mathrm{H}$, s), $0.03(3 \mathrm{H}, \mathrm{s}), 0.03(3 \mathrm{H}, \mathrm{s}), 0.00(3 \mathrm{H}, \mathrm{s}) ;{ }^{13} \mathrm{C}-\mathrm{NMR}\left(100 \mathrm{MHz}, \mathrm{CDCl}_{3}\right) \delta \mathrm{ppm}: 150.2,114.1,79.1$, 66.3, 49.5, 35.7, 30.0, 26.1, 26.0, 23.9, 22.5, 18.4, 18.3, 14.7, -3.9, -5.0, -5.3; HRESIMS (m/z) calcd. for $\mathrm{C}_{22} \mathrm{H}_{46} \mathrm{O}_{2} \mathrm{Si}_{2} \mathrm{Na}(\mathrm{M}+\mathrm{Na})^{+} 421.2934$, found 421.2914; Anal. Calcd. for $\mathrm{C}_{22} \mathrm{H}_{46} \mathrm{O}_{2} \mathrm{Si}_{2}$ : C, 66.26; $\mathrm{H}$, 11.63. Found: $\mathrm{C}, 66.36 ; \mathrm{H}, 11.50$.

\section{8. (S)-1-\{(1R,2S)-2-[(R)-2,2-Dimethyl-[1,3]dioxolan-4-yl]-2-methylcyclopentyl\}ethanol (9)}

To a solution of E-olefin 7 (3.86 g, $9.69 \mathrm{mmol})$ in THF $(19.4 \mathrm{~mL})$ was added catecholborane $(6.40 \mathrm{~mL}, 60.1 \mathrm{mmol})$ dropwise at $0{ }^{\circ} \mathrm{C}$. After stirring for $12 \mathrm{hr}$ at the same temperature, $1 \mathrm{M} \mathrm{NaOH}$ solution $(12.9 \mathrm{~mL})$ and $35 \%$ aqueous $\mathrm{H}_{2} \mathrm{O}_{2}$ solution $(36.9 \mathrm{~mL})$ were added to the mixture at r.t. After stirring for $2 \mathrm{hr}$, the resultant mixture was diluted with $\mathrm{CHCl}_{3}$, washed with $\mathrm{H}_{2} \mathrm{O}$ and brine, dried and then concentrated to afford a mixture of diol 8 and triol 8a. To a solution of the crude alcohols in acetone $(96.9 \mathrm{~mL})$ was added $p-\mathrm{TsOH} \cdot \mathrm{H}_{2} \mathrm{O}(735 \mathrm{mg}, 3.88 \mathrm{mmol})$ at r.t. After stirring for $2 \mathrm{hr}$ at the same temperature, the mixture was diluted with $\mathrm{Et}_{2} \mathrm{O}$, washed with saturated aqueous $\mathrm{NaHCO}_{3}$ solution, $\mathrm{H}_{2} \mathrm{O}$ and brine, and then dried. Removal of the solvent gave a residue which was then purified by silica gel column chromatography (hexane/acetone $=4: 1)$ to generate acetonide $9(2.11 \mathrm{~g}$, $95 \%$ yield) as a colorless oil. $[\alpha]_{\mathrm{D}}{ }^{25}+4.0\left(\right.$ c $\left.0.58, \mathrm{CHCl}_{3}\right)$; IR (neat) $3443,2956 \mathrm{~cm}^{-1} ;{ }^{1} \mathrm{H}-\mathrm{NMR}(400$ $\left.\mathrm{MHz}, \mathrm{CDCl}_{3}\right) \delta$ ppm: $4.42(1 \mathrm{H}, \mathrm{dd}, J=8.5,6.5 \mathrm{~Hz}), 4.01(1 \mathrm{H}, \mathrm{m}), 3.96(1 \mathrm{H}, \mathrm{dd}, J=7.8,6.5 \mathrm{~Hz}), 3.66$ $(1 \mathrm{H}, \mathrm{t}, J=8.2 \mathrm{~Hz}), 2.07(1 \mathrm{H}, \mathrm{s}), 1.89(1 \mathrm{H}, \mathrm{m}), 1.69-1.50(4 \mathrm{H}, \mathrm{m}), 1.41(3 \mathrm{H}, \mathrm{s}), 1.35(3 \mathrm{H}, \mathrm{s}), 1.37-1.33$ $(2 \mathrm{H}, \mathrm{m}), 1.20(3 \mathrm{H}, \mathrm{d}, J=6.2 \mathrm{~Hz}), 1.07(3 \mathrm{H}, \mathrm{s}),{ }^{13} \mathrm{C}-\mathrm{NMR}\left(100 \mathrm{MHz}, \mathrm{CDCl}_{3}\right) \delta \mathrm{ppm}: 108.5,79.9,69.3$, 
66.9, 59.1, 45.1, 35.0, 31.2, 27.8, 26.5, 25.3, 24.2, 23.3; HRESIMS (m/z) calcd. for $\mathrm{C}_{13} \mathrm{H}_{25} \mathrm{O}_{3}(\mathrm{M}+\mathrm{H})^{+}$ 229.1804, found 229.1810; Anal. Calcd. for $\mathrm{C}_{13} \mathrm{H}_{24} \mathrm{O}_{3}$ : C, 68.38; H, 10.59. Found: C, 68.43; H, 10.59 .

3.9. (R)-1-[(1S,2R)-2-((S)-1-Hydroxyethyl)-1-methylcyclopentyl]but-3-en-1-ol (11a) and (S)-1[(1S,2R)-2-((S)-1-hydroxyethyl)-1-methylcyclopentyl] but-3-en-1-ol (11b)

To a solution of acetonide $9(2.11 \mathrm{~g}, 9.24 \mathrm{mmol})$ in THF was added a solution of $\mathrm{HIO}_{4} \cdot 2 \mathrm{H}_{2} \mathrm{O}$ (12.6 g, $55.4 \mathrm{mmol})$ in $\mathrm{H}_{2} \mathrm{O}(93.0 \mathrm{~mL})$ at r.t. After stirring for $3 \mathrm{hr}$ at $45^{\circ} \mathrm{C}$, the mixture was diluted with $\mathrm{Et}_{2} \mathrm{O}$, washed with $\mathrm{H}_{2} \mathrm{O}$ and brine, dried and then concentrated to afford crude hemiacetal 10. To a solution of crude hemiacetal 10 in $\mathrm{Et}_{2} \mathrm{O}(93.0 \mathrm{~mL})$ was added allyl magnesium bromide $(33.0 \mathrm{~mL}$, $32.3 \mathrm{mmol}, 1.0 \mathrm{M}$ in $\mathrm{Et}_{2} \mathrm{O}$ solution) at $-78{ }^{\circ} \mathrm{C}$. After stirring for $1 \mathrm{hr}$ at $0{ }^{\circ} \mathrm{C}$, the mixture was diluted with $\mathrm{Et}_{2} \mathrm{O}$, washed with saturated aqueous $\mathrm{NH}_{4} \mathrm{Cl}$ solution, $\mathrm{H}_{2} \mathrm{O}$ and brine, and then dried. Removal of the solvent gave a residue which was then purified by silica gel column chromatography (hexane/AcOEt $=8: 1)$ to generate diol 11a $(914 \mathrm{mg}, 50 \%$ yield) as a colorless oil and diol 11b (650 mg, $36 \%$ yield) as a white solid. Compound 11a: $[\alpha]_{\mathrm{D}}{ }^{25}-5.0\left(c \mathrm{c} 0.39, \mathrm{CHCl}_{3}\right)$; IR (neat) 3306, $2955 \mathrm{~cm}^{-1}$; ${ }^{1} \mathrm{H}-\mathrm{NMR}\left(400 \mathrm{MHz}, \mathrm{CDCl}_{3}\right) \delta \mathrm{ppm}: 5.87(1 \mathrm{H}, \mathrm{m}), 5.19(1 \mathrm{H}, \mathrm{d}, J=10.4 \mathrm{~Hz}), 5.18(1 \mathrm{H}, \mathrm{d}$, $J=16.8 \mathrm{~Hz}), 3.86(1 \mathrm{H}, \mathrm{m}), 3.68(1 \mathrm{H}, \mathrm{dd}, J=10.5,2.3 \mathrm{~Hz}), 3.18(2 \mathrm{H}, \mathrm{br} \mathrm{s}), 2.34(1 \mathrm{H}, \mathrm{m}), 2.13(1 \mathrm{H}, \mathrm{m})$, $1.80(1 \mathrm{H}, \mathrm{m}), 1.62-1.34(5 \mathrm{H}, \mathrm{m}), 1.22(1 \mathrm{H}, \mathrm{m}), 1.17(3 \mathrm{H}, \mathrm{d}, J=6.2 \mathrm{~Hz}), 1.09(3 \mathrm{H}, \mathrm{s}) ;{ }^{13} \mathrm{C}-\mathrm{NMR}$ $\left(100 \mathrm{MHz}, \mathrm{CDCl}_{3}\right) \delta$ ppm: 136.1, 118.7, 72.5, 69.2, 58.8, 47.8, 39.5, 37.1, 30.2, 22.7, 22.5, 22.4; HRESIMS (m/z) calcd. for $\mathrm{C}_{12} \mathrm{H}_{23} \mathrm{O}_{2}(\mathrm{M}+\mathrm{H})^{+} 199.1698$, found 199.1713; Anal. Calcd. for $\mathrm{C}_{12} \mathrm{H}_{22} \mathrm{O}_{2}$ : C, 72.68; H, 11.18. Found: C, 72.40; H, 10.99. Compound 11b: mp 93-95 ${ }^{\circ} \mathrm{C} ;[\alpha]_{\mathrm{D}}{ }^{25}-35.0$ (c 0.20 , $\mathrm{CHCl}_{3}$ ); IR (KBr) 3351, $2953 \mathrm{~cm}^{-1} ;{ }^{1} \mathrm{H}-\mathrm{NMR}\left(400 \mathrm{MHz}, \mathrm{CDCl}_{3}\right) \delta$ ppm: $5.30(1 \mathrm{H}, \mathrm{m}), 5.18(1 \mathrm{H}, \mathrm{d}$, $J=10.2 \mathrm{~Hz}), 5.17(1 \mathrm{H}, \mathrm{d}, J=16.9 \mathrm{~Hz}), 4.11(1 \mathrm{H}, \mathrm{m}), 3.76(1 \mathrm{H}, \mathrm{dd}, J=10.8,2.1 \mathrm{~Hz}), 2.48(1 \mathrm{H}, \mathrm{m})$, $2.27(2 \mathrm{H}$, br s), $2.13(1 \mathrm{H}, \mathrm{m}), 1.85(1 \mathrm{H}, \mathrm{m}), 1.67(2 \mathrm{H}, \mathrm{m}), 1.60(2 \mathrm{H}, \mathrm{m}), 1.45(2 \mathrm{H}, \mathrm{m}),, 1.22(3 \mathrm{H}, \mathrm{d}$, $J=6.2 \mathrm{~Hz}), 1.19(3 \mathrm{H}, \mathrm{s}) ;{ }^{13} \mathrm{C}-\mathrm{NMR}\left(100 \mathrm{MHz}, \mathrm{CDCl}_{3}\right) \delta \mathrm{ppm:} \mathrm{136.2,} \mathrm{118.6,} \mathrm{75.7,} \mathrm{69.5,} \mathrm{59.5,} \mathrm{47.5,}$ 37.8, 36.5, 32.0, 29.5, 23.9, 23.4; HRESIMS $(\mathrm{m} / \mathrm{z})$ calcd. for $\mathrm{C}_{12} \mathrm{H}_{23} \mathrm{O}_{2}(\mathrm{M}+\mathrm{H})^{+} 199.1698$, found 199.1681; Anal. Calcd. for $\mathrm{C}_{12} \mathrm{H}_{22} \mathrm{O}_{2}$ : C, 72.68; H, 11.18. Found: C, 72.65; H, 10.91 .

\subsection{0. (1R,2R,3aS,3R)-3-Allyl-1,3a-dimethylhexahydrocyclopenta[c]furan (12a)}

To a solution of diol 11a $(34.4 \mathrm{mg}, 0.174 \mathrm{mmol})$ in $\mathrm{CH}_{2} \mathrm{Cl}_{2}(1.7 \mathrm{~mL})$ were added $\mathrm{Et}_{3} \mathrm{~N}(105 \mathrm{mg}$, $1.04 \mathrm{mmol}$ ), DMAP (106 mg, $0.868 \mathrm{mmol})$ and $p$-toluenesulfonyl chloride (132 $\mathrm{mg}, 0.694 \mathrm{mmol})$ at r.t. The mixture was stirred for two days at the same temperature. The mixture was diluted with $\mathrm{Et}_{2} \mathrm{O}$, washed with saturated aqueous $\mathrm{NH}_{4} \mathrm{Cl}$ solution, $\mathrm{H}_{2} \mathrm{O}$ and brine, and then dried. Removal of the solvent gave a residue which was then purified by silica gel column chromatography (hexane/AcOEt $=10: 1$ ) to generate tetrahydrofuran $12 \mathrm{a}(24.1 \mathrm{mg}, 77 \%$ yield $)$ as a colorless oil. $[\alpha]_{\mathrm{D}}{ }^{26}+21.5\left(\mathrm{c} 1.58, \mathrm{CHCl}_{3}\right)$; IR (neat) 2953, $2870 \mathrm{~cm}^{-1}$; ${ }^{1} \mathrm{H}-\mathrm{NMR}\left(400 \mathrm{MHz}, \mathrm{CDCl}_{3}\right) \delta \mathrm{ppm}: 5.84(1 \mathrm{H}, \mathrm{m}), 5.09(1 \mathrm{H}, \mathrm{m}), 5.03(1 \mathrm{H}$, m), $4.25(1 \mathrm{H}$, quint., $J=6.5 \mathrm{~Hz}), 3.64(1 \mathrm{H}, \mathrm{dd}, J=9.0,4.8 \mathrm{~Hz}), 2.30-2.15(2 \mathrm{H}, \mathrm{m}), 2.07(1 \mathrm{H}, \mathrm{m})$, 1.68-1.59 (5H, m), $1.46(1 \mathrm{H}, \mathrm{m}), 1.16(3 \mathrm{H}, \mathrm{d}, J=6.5 \mathrm{~Hz}), 1.07(3 \mathrm{H}, \mathrm{s})$; NOESY correlations $(\mathrm{H} / \mathrm{H})$ : H-1/H-4; H-3/H-17; H-9/H-17; ${ }^{13} \mathrm{C}-\mathrm{NMR}\left(100 \mathrm{MHz}, \mathrm{CDCl}_{3}\right) \delta \mathrm{ppm:} \mathrm{136.7,} \mathrm{116.4,} \mathrm{85.0,} \mathrm{74.5,} \mathrm{57.0,}$ 39.1, 35.4, 27.3, 26.5, 22.1, 17.3; HRESIMS $(\mathrm{m} / \mathrm{z})$ calcd. for $\mathrm{C}_{12} \mathrm{H}_{21} \mathrm{O}(\mathrm{M}+\mathrm{H})^{+} 181.1592$, found 181.1590; Anal. Calcd. for $\mathrm{C}_{12} \mathrm{H}_{20} \mathrm{O}$ : C, 79.94; H, 11.18. Found: C, 80.11; H, 11.10. 


\subsection{1. (1R,2R,3aS,3S)-3-Allyl-1,3a-dimethylhexahydrocyclopenta[c]furan (12b)}

To a solution of diol $11 \mathrm{~b}(32.4 \mathrm{mg}, 0.163 \mathrm{mmol})$ in $\mathrm{CH}_{2} \mathrm{Cl}_{2}(1.6 \mathrm{~mL})$ were added $\mathrm{Et}_{3} \mathrm{~N}(82.8 \mathrm{mg}$, $0.817 \mathrm{mmol})$, DMAP (79.9 mg, $0.654 \mathrm{mmol})$ and $p$-toluenesulfonyl chloride $(93.5 \mathrm{mg}, 0.490 \mathrm{mmol})$ at r.t. The mixture was stirred for 2 days at the same temperature. The mixture was diluted with $\mathrm{Et}_{2} \mathrm{O}$, washed with saturated aqueous $\mathrm{NH}_{4} \mathrm{Cl}$ solution, $\mathrm{H}_{2} \mathrm{O}$ and brine, and then dried. Removal of the solvent gave a residue which was then purified by silica gel column chromatography (hexane/AcOEt $=5: 1)$ to generate tetrahydrofuran 12b $\left(27.1 \mathrm{mg}, 93 \%\right.$ yield) as a colorless oil. $[\alpha]_{\mathrm{D}}{ }^{26}-35.1$ (c 1.14, $\left.\mathrm{CHCl}_{3}\right)$; IR (neat) 2951, $2866 \mathrm{~cm}^{-1} ;{ }^{1} \mathrm{H}-\mathrm{NMR}\left(400 \mathrm{MHz}, \mathrm{CDCl}_{3}\right) \delta \mathrm{ppm}: 5.87(1 \mathrm{H}, \mathrm{m}), 5.12(1 \mathrm{H}, \mathrm{m}), 5.04(1 \mathrm{H}, \mathrm{m})$, $3.84(1 \mathrm{H}$, quint., $J=6.4 \mathrm{~Hz}), 3.32(1 \mathrm{H}, \mathrm{dd}, J=8.4,4.9 \mathrm{~Hz}), 2.36-2.23(2 \mathrm{H}, \mathrm{m}), 1.97(1 \mathrm{H}, \mathrm{m})$, 1.63-1.59 (6H, m), $1.19(3 \mathrm{H}, \mathrm{d}, J=6.5 \mathrm{~Hz}), 1.07(3 \mathrm{H}, \mathrm{s})$; NOESY correlations $(\mathrm{H} / \mathrm{H}): \mathrm{H}-1 / \mathrm{H}-4$; $\mathrm{H}-2 / \mathrm{H}-3$; H-2/H-8; H-3/H-17; H-8/H-17; ${ }^{13} \mathrm{C}-\mathrm{NMR}\left(100 \mathrm{MHz}, \mathrm{CDCl}_{3}\right) \delta$ ppm: 136.1, 116.3, 86.9, 75.4, 56.1, 35.1, 34.7, 30.0, 27.7, 26.8, 25.9, 15.5; HRESIMS $(\mathrm{m} / \mathrm{z})$ calcd. for $\mathrm{C}_{12} \mathrm{H}_{21} \mathrm{O}(\mathrm{M}+\mathrm{H})^{+}$ 181.1592, found 181.1577; Anal. Calcd. for $\mathrm{C}_{12} \mathrm{H}_{20} \mathrm{O}$ : C, 79.94; H, 11.18. Found: C, 79.91; H, 11.14.

\subsection{Conversion from diol 11b to diol 11a}

To a solution of diol $11 b(50.0 \mathrm{mg}, 0.252 \mathrm{mmol})$ in DMF $(252 \mu \mathrm{L})$ were added imidazole $(21.0 \mathrm{mg}$, $0.308 \mathrm{mmol})$ and TBDPSCl $(83.5 \mathrm{mg}, 0.304 \mathrm{mmol})$ and the mixture was stirred at r.t. for $1 \mathrm{hr}$. The mixture was diluted with $\mathrm{Et}_{2} \mathrm{O}$, washed with saturated aqueous $\mathrm{NaHCO}_{3}$ solution, $\mathrm{H}_{2} \mathrm{O}$ and brine and then dried. The crude mixture was diluted with $\mathrm{Et}_{2} \mathrm{O}$ and filtered through a silica gel pad. The filtrate was concentrated to afford the crude alcohol. To a solution of the crude alcohol in $\mathrm{CH}_{3} \mathrm{CN}(2.5 \mathrm{~mL})$ was added IBX (212 mg, $0.757 \mathrm{mmol})$ at r.t. After stirring for $30 \mathrm{~min}$ at $80^{\circ} \mathrm{C}$, the mixture was diluted with $\mathrm{Et}_{2} \mathrm{O}$ and then filtered through a Celite pad. Removal of the solvent gave a residue which was filtered through a silica gel pad to afford the crude ketone. To a solution of the crude ketone in $\mathrm{MeOH}$ $(2.5 \mathrm{~mL})$ was added $\mathrm{NaBH}_{4}(28.6 \mathrm{mg}, 0.756 \mathrm{mmol})$ at r.t. After stirring for $2 \mathrm{hr}$ under reflux, the mixture was diluted with $\mathrm{Et}_{2} \mathrm{O}$, washed with $\mathrm{H}_{2} \mathrm{O}$ and brine and then dried. Removal of the solvent gave a residue which was then filtered through a silica gel pad to afford the crude alcohols. To a solution of the crude alcohols in THF $(2.5 \mathrm{~mL})$ was added TBAF $(760 \mu \mathrm{L}, 0.760 \mathrm{mmol}, 1.0 \mathrm{M}$ in THF solution) at r.t. After stirring for $12 \mathrm{hr}$ at $40{ }^{\circ} \mathrm{C}$, the mixture was diluted with $\mathrm{Et}_{2} \mathrm{O}$, washed with $\mathrm{H}_{2} \mathrm{O}$ and brine and then dried. Removal of the solvent gave a residue which was then purified by silica gel column chromatography (hexane/AcOEt $=4: 1$ ) to generate diol 11a $(34.6 \mathrm{mg}, 69 \%)$ and diol 11b (6.9 mg, $14 \%)$.

\subsection{3. (1S,2R)-1-[(R)-1-(tert-Butyldimethylsiloxy)but-3-enyl]-2-[(S)-1-(tert-butyldimethylsiloxy)ethyl]- 1-methylcyclopentane (13)}

To a solution of diol 11a (988 mg, $4.98 \mathrm{mmol})$ in $\mathrm{CH}_{2} \mathrm{Cl}_{2}(2.7 \mathrm{~mL})$ were added 2,6-lutidine (2.67 g, $24.9 \mathrm{mmol})$ and TBSOTf $(3.95 \mathrm{~g}, 14.9 \mathrm{mmol})$ and the mixture was stirred at $0{ }^{\circ} \mathrm{C}$ for $30 \mathrm{~min}$. The mixture was diluted with $\mathrm{Et}_{2} \mathrm{O}$, washed with saturated aqueous $\mathrm{NaHCO}_{3}$ solution, $\mathrm{H}_{2} \mathrm{O}$ and brine, and then dried. Removal of the solvent gave a residue which was then purified by silica gel column chromatography (hexane only) to generate bis-silyl ether $\mathbf{1 3}(2.11 \mathrm{~g}, 99 \%$ yield) as a colorless oil. 
$[\alpha]_{\mathrm{D}}{ }^{25}+0.97\left(\right.$ c 1.07, $\left.\mathrm{CHCl}_{3}\right)$; IR (neat) 2956, 2885, $1471 \mathrm{~cm}^{-1} ;{ }^{1} \mathrm{H}-\mathrm{NMR}\left(400 \mathrm{MHz}, \mathrm{CDCl}_{3}\right) \delta \mathrm{ppm}$ : $5.90(1 \mathrm{H}, \mathrm{m}), 5.00(2 \mathrm{H}, \mathrm{m}), 4.13(1 \mathrm{H}$, quint, $J=6.1 \mathrm{~Hz}), 3.80(1 \mathrm{H}, \mathrm{dd}, J=6.5,3.7 \mathrm{~Hz}), 2.38(1 \mathrm{H}, \mathrm{m})$, $2.22(1 \mathrm{H}, \mathrm{m}), 1.85-1.57(6 \mathrm{H}, \mathrm{m}), 1.19(1 \mathrm{H}, \mathrm{m}), 1.09(3 \mathrm{H}, \mathrm{d}, J=6.1 \mathrm{~Hz}), 1.04(3 \mathrm{H}, \mathrm{s}), 0.89(9 \mathrm{H}, \mathrm{s})$, $0.88(9 \mathrm{H}, \mathrm{s}), 0.06(12 \mathrm{H}, \mathrm{m}) ;{ }^{13} \mathrm{C}-\mathrm{NMR}\left(100 \mathrm{MHz}, \mathrm{CDCl}_{3}\right) \delta \mathrm{ppm}: 137.7,115.8,77.2,76.8,69.1,56.6$, 50.1, 40.3, 35.1, 27.4, 27.1, 26.2, 26.0, 22.5, 18.4, 18.1, -3.0, -3.4, -3.6, -4.0; HRESIMS (m/z) calcd. for $\mathrm{C}_{24} \mathrm{H}_{51} \mathrm{O}_{2} \mathrm{Si}_{2}(\mathrm{M}+\mathrm{H})^{+}$427.3428, found 427.3437; Anal. Calcd. for $\mathrm{C}_{24} \mathrm{H}_{50} \mathrm{O}_{2} \mathrm{Si}_{2}: \mathrm{C}, 67.54 ; \mathrm{H}, 11.81$. Found: C, 67.44; H, 11.66.

\subsection{4. (R)-3-(tert-Butyldimethylsiloxy)-3-\{(1S,2R)-2-[(S)-1-(tert-butyldimethylsiloxy)ethyl]-1-methyl-} cyclopentyl \}propionaldehyde (14)

A cold $\left(-78^{\circ} \mathrm{C}\right)$ solution of bis-silyl ether $13(482 \mathrm{mg}, 1.13 \mathrm{mmol})$ in $\mathrm{CH}_{2} \mathrm{Cl}_{2}(56.5 \mathrm{~mL})$ was treated with ozone until the blue color generated persisted for more than $15 \mathrm{~min}$. Excess ozone was removed using an argon flow. To the mixture were then added $\mathrm{MeOH}(56.5 \mathrm{~mL}), \mathrm{Zn}$ powder $(739 \mathrm{mg}$, $11.3 \mathrm{mmol})$, KI (1.88 g, $11.3 \mathrm{mmol})$ and $\mathrm{AcOH}(682 \mathrm{mg}, 11.4 \mathrm{mmol})$. The mixture was allowed to warm to r.t., stirred for $1 \mathrm{hr}$ at the same temperature and then concentrated under reduced pressure. The resultant residue was diluted with $\mathrm{Et}_{2} \mathrm{O}$, washed with saturated aqueous $\mathrm{NaHCO}_{3}$ solution, $\mathrm{H}_{2} \mathrm{O}$ and brine and then dried. Removal of the solvent gave a residue which was then purified by silica gel column chromatography (hexane/AcOEt $=20: 1$ ) to generate aldehyde 14 (484 mg, quantitative yield) as a colorless oil. $[\alpha]_{\mathrm{D}}{ }^{25}-0.56\left(c\right.$ 1.08, $\left.\mathrm{CHCl}_{3}\right)$; IR (neat) 2955, 2857, $1727 \mathrm{~cm}^{-1} ;{ }^{1} \mathrm{H}-\mathrm{NMR}(400 \mathrm{MHz}$, $\left.\mathrm{CDCl}_{3}\right) \delta$ ppm: $9.85(1 \mathrm{H}, \mathrm{dd}, J=2.7,1.3 \mathrm{~Hz}), 4.42(1 \mathrm{H}, \mathrm{dd}, J=6.0,4.1 \mathrm{~Hz}), 4.10$ (1H, quint, $J=6.1 \mathrm{~Hz}), 2.67(2 \mathrm{H}, \mathrm{m}), 1.84(1 \mathrm{H}, \mathrm{m}), 1.74(2 \mathrm{H}, \mathrm{m}), 1.61(3 \mathrm{H}, \mathrm{m}), 1.23(1 \mathrm{H}, \mathrm{m}), 1.12(3 \mathrm{H}, \mathrm{d}$, $J=6.1 \mathrm{~Hz}), 1.05(3 \mathrm{H}, \mathrm{s}), 0.88(9 \mathrm{H}, \mathrm{s}), 0.88(9 \mathrm{H}, \mathrm{s}), 0.06(12 \mathrm{H}, \mathrm{m}) ;{ }^{13} \mathrm{C}-\mathrm{NMR}\left(100 \mathrm{MHz}, \mathrm{CDCl}_{3}\right) \delta$ ppm: 201.9, 70.9, 69.0, 56.3, 50.5, 49.6, 35.4, 27.3, 26.7, 26.0, 22.4, 22.3, -3.4, -3.7, -4.0, -4.1; HRESIMS $(\mathrm{m} / \mathrm{z})$ calcd. for $\mathrm{C}_{23} \mathrm{H}_{48} \mathrm{O}_{3} \mathrm{Si}_{2} \mathrm{Na}(\mathrm{M}+\mathrm{Na})^{+}$451.3040, found 451.3052; Anal. Calcd. for $\mathrm{C}_{23} \mathrm{H}_{48} \mathrm{O}_{3} \mathrm{Si}_{2}$ : C, 64.42; H, 11.28. Found: C, 64.40; H, 11.10.

\subsection{5. (6E,9R)-9-(tert-Butyldimethylsiloxy)-9-\{(1R,2S)-2-[(S)-1-(tert-butyldimethylsiloxy)ethyl]-1-} methylcyclopentyl\}-2,6-dimethylnona-2,6-dien-5-one (16)

To a solution of phosphonate 15 [5] (306 mg, $1.17 \mathrm{mmol})$ in THF (700 $\mu \mathrm{L})$ was added ${ }^{n} \mathrm{BuLi}$ $\left(591 \mu \mathrm{L}, 0.935 \mathrm{mmol}, 1.58 \mathrm{M}\right.$ in hexane solution) at $0{ }^{\circ} \mathrm{C}$. The mixture was stirred for $1 \mathrm{hr}$ at the same temperature and a solution of aldehyde $14(200 \mathrm{mg}, 0.467 \mathrm{mmol})$ in THF $(4.0 \mathrm{~mL})$ was added dropwise at r.t. After stirring for $5 \mathrm{hr}$, the mixture was diluted with $\mathrm{Et}_{2} \mathrm{O}$, washed with saturated aqueous $\mathrm{NH}_{4} \mathrm{Cl}$ solution, $\mathrm{H}_{2} \mathrm{O}$ and brine, and then dried. Removal of the solvent gave a residue which was then purified by silica gel column chromatography (hexane/benzene $=4: 1$ ) to generate $\alpha, \beta$-unsaturated ketone 16 (123 mg, 49\% yield) as a white solid and recovered aldehyde 14 (36.5 mg, 18\% yield). Compound 16: $\mathrm{mp} 55-58{ }^{\circ} \mathrm{C} ;[\alpha]_{\mathrm{D}}{ }^{25}+11.2\left(c\right.$ 0.57, $\left.\mathrm{CHCl}_{3}\right)$; UV (MeOH) $\lambda_{\max }(\varepsilon) \mathrm{nm}: 234$ (18800); IR (KBr) 2956, 2931, 2856, $1674 \mathrm{~cm}^{-1} ;{ }^{1} \mathrm{H}-\mathrm{NMR}\left(400 \mathrm{MHz}, \mathrm{CDCl}_{3}\right) \delta$ ppm: $6.83(1 \mathrm{H}, \mathrm{t}$, $J=6.1 \mathrm{~Hz}), 5.34(1 \mathrm{H}, \mathrm{m}), 4.10(1 \mathrm{H}$, quint, $J=6.2 \mathrm{~Hz}), 3.97(1 \mathrm{H}, \mathrm{t}, J=5.6 \mathrm{~Hz}), 3.38(2 \mathrm{H}, \mathrm{d}$, $J=7.0 \mathrm{~Hz}), 2.49(2 \mathrm{H}, \mathrm{m}), 1.85(1 \mathrm{H}, \mathrm{m}), 1.78(3 \mathrm{H}, \mathrm{s}), 1.74(3 \mathrm{H}, \mathrm{s}), 1.80-1.74(2 \mathrm{H}, \mathrm{m}), 1.64(3 \mathrm{H}, \mathrm{s})$, 1.64-1.57 (4H, m), $1.11(3 \mathrm{H}, \mathrm{d}, J=6.1 \mathrm{~Hz}), 1.06(3 \mathrm{H}, \mathrm{s}), 0.90(9 \mathrm{H}, \mathrm{s}), 0.88(9 \mathrm{H}, \mathrm{s}), 0.08(6 \mathrm{H}, \mathrm{d}$, 
$J=12.6 \mathrm{~Hz}), 0.06(6 \mathrm{H}, \mathrm{d}, J=6.5 \mathrm{~Hz}) ;{ }^{13} \mathrm{C}-\mathrm{NMR}\left(100 \mathrm{MHz}, \mathrm{CDCl}_{3}\right) \delta \mathrm{ppm:} \mathrm{199.9,} \mathrm{141.5,} \mathrm{136.9,}$ 134.6, 117.1, 77.2, 75.9, 69.1, 56.5, 50.1, 37.1, 35.6, 35.1, 27.2, 27.1, 26.1, 26.0, 25.7, 22.5, 22.4, 18.3, 18.1, 11.9, -3.2, -3.4, -3.8, -3.9; HRESIMS (m/z) calcd. for $\mathrm{C}_{31} \mathrm{H}_{61} \mathrm{O}_{3} \mathrm{Si}_{2}(\mathrm{M}+\mathrm{H})^{+}$537.4159, found 537.4168; Anal. Calcd. for $\mathrm{C}_{31} \mathrm{H}_{60} \mathrm{O}_{3} \mathrm{Si}_{2}$ : C, 69.34; H, 11.26. Found: C, 69.40; H, 11.05.

3.16. (5R,6E,9R)-9-(tert-Butyldimethylsiloxy)-9-\{(1R,2S)-2-[(S)-1-(tert-butyldimethylsiloxy)ethyl]-1methylcyclopentyl\}-2,6-dimethylnona-2,6-dien-5-ol and (5S,6E,9R)-9-(tert-butyldimethylsiloxy)-9$\{(1 R, 2 S)-2-[(S)-1$-(tert-butyldimethylsiloxy)ethyl]-1-methylcyclopentyl\}-2,6-dimethylnona-2,6-dien-5ol (17)

To a solution of $\mathrm{CeCl}_{3} \cdot 7 \mathrm{H}_{2} \mathrm{O}(144 \mathrm{mg}, 0.386 \mathrm{mmol})$ in $\mathrm{MeOH}(9.3 \mathrm{~mL})$ was added $\mathrm{NaBH}_{4}$ (11.0 mg, $0.290 \mathrm{mmol}$ ) at $0{ }^{\circ} \mathrm{C}$. The mixture was then added to a solution of $\alpha, \beta$-unsaturated ketone 16 (104 mg, $0.193 \mathrm{mmol})$ in $\mathrm{MeOH}(10.0 \mathrm{~mL})$ at $0{ }^{\circ} \mathrm{C}$ and stirred for $30 \mathrm{~min}$ at the same temperature. The mixture was diluted with $\mathrm{Et}_{2} \mathrm{O}$, washed with $\mathrm{H}_{2} \mathrm{O}$ and brine, and then dried. Removal of the solvent gave a residue which was then purified by silica gel column chromatography $\left(\mathrm{CHCl}_{3}\right.$ only) to generate a diastereomeric mixture of allylic alcohol 17 (103 mg, 99\% yield) as a colorless oil. IR (neat) 3353, $2957 \mathrm{~cm}^{-1}$; ${ }^{1} \mathrm{H}-\mathrm{NMR}\left(400 \mathrm{MHz}, \mathrm{CDCl}_{3}\right) \delta$ ppm: $5.54(1 \mathrm{H}, \mathrm{m}), 5.11(1 \mathrm{H}, \mathrm{m}), 4.14(1 \mathrm{H}, \mathrm{m}), 4.00(1 \mathrm{H}$, $\mathrm{m}), 3.80(1 \mathrm{H}, \mathrm{m}), 2.42-2.14(4 \mathrm{H}, \mathrm{m}), 1.86(1 \mathrm{H}, \mathrm{m}), 1.80-1.57(5 \mathrm{H}, \mathrm{m}), 1.72(3 \mathrm{H}, \mathrm{s}), 1.64(3 \mathrm{H}, \mathrm{s}), 1.62$ $(3 \mathrm{H}, \mathrm{s}), 1.15(1 \mathrm{H}, \mathrm{m}), 1.09(3 \mathrm{H}, \mathrm{d}, J=6.1 \mathrm{~Hz}), 1.02(1.5 \mathrm{H}, \mathrm{s}), 1.02(1.5 \mathrm{H}, \mathrm{s}), 0.88(9 \mathrm{H}, \mathrm{d}, J=6.9 \mathrm{~Hz})$, $0.88(9 \mathrm{H}, \mathrm{d}, J=5.4 \mathrm{~Hz}), 0.06(12 \mathrm{H}, \mathrm{m}) ;{ }^{13} \mathrm{C}-\mathrm{NMR}\left(100 \mathrm{MHz}, \mathrm{CDCl}_{3}\right) \delta \mathrm{ppm}: 136.6,136.5,134.8$, 134.7, 125.6, 125.5, 120.2, 120.2, 77.2, 77.2, 68.9, 68.9, 56.2, 56.2, 50.2, 50.2, 35.1, 35.0, 34.1, 34.0, 26.7, 26.6, 26.5, 26.3, 26.2, 26.0, 25.9, 22.5, 22.1, 22.1, 22.1, 18.4, 18.1, 18.0, 12.1, 12.1, -3.1, -3.2, $-3.4,-3.5,-3.7,-3.7,-4.0,-4.0$; HRESIMS $(\mathrm{m} / \mathrm{z})$ calcd. for $\mathrm{C}_{31} \mathrm{H}_{62} \mathrm{O}_{3} \mathrm{Si}_{2} \mathrm{Na}(\mathrm{M}+\mathrm{Na})^{+} 561.4135$, found 561.4156; Anal. Calcd. for $\mathrm{C}_{31} \mathrm{H}_{62} \mathrm{O}_{3} \mathrm{Si}_{2}$ : C, 69.08; H, 11.59. Found: C, 68.92; H, 11.30.

3.17. (1R,3E,5R)-1-[(1S,2R)-2-((S)-1-Hydroxyethyl)-1-methylcyclopentyl]-4,8-dimethyl-5-trityloxynona-3,7-dien-1-ol and (1R,3E,5S)-1-[(1S,2R)-2-((S)-1-hydroxyethyl)-1-methylcyclopentyl]-4,8dimethyl-5-trityloxynona-3,7-dien-1-ol (18)

To a solution of the diastereomeric mixture of allylic alcohol 17 (40.0 mg, $0.074 \mathrm{mmol})$ in pyridine $(740 \mu \mathrm{L})$ were added DMAP $(5.0 \mathrm{mg}, 0.041 \mathrm{mmol})$ and $\mathrm{TrCl}(103 \mathrm{mg}, 0.395 \mathrm{mmol})$ at r.t. After stirring for 4 days at $80{ }^{\circ} \mathrm{C}$, the mixture was diluted with $\mathrm{Et}_{2} \mathrm{O}$, washed with $\mathrm{H}_{2} \mathrm{O}$ and brine, and then dried. Removal of the solvent gave a residue which was filtered through a short-path silica gel pad (hexane/AcOEt $=20: 1$ ). The filtrate was then concentrated to afford the crude trityl ether. To a solution of the crude trityl ether in DMF $(1.5 \mathrm{~mL})$ was added TBAF $(1.5 \mathrm{~mL}, 0.150 \mathrm{mmol}, 1.0 \mathrm{M}$ in THF solution) at r.t. After stirring for 2 days at $50{ }^{\circ} \mathrm{C}$, the mixture was diluted with $\mathrm{Et}_{2} \mathrm{O}$, washed with $\mathrm{H}_{2} \mathrm{O}$ and brine and then dried. Removal of the solvent gave a residue which was then purified by silica gel column chromatography (hexane/AcOEt $=10: 1$ ) to generate a diastereomeric mixture of diol 18 (40.9 mg, quantitative yield) as a colorless oil. IR (neat) 3344, $2961 \mathrm{~cm}^{-1} ;{ }^{1} \mathrm{H}-\mathrm{NMR}\left(400 \mathrm{MHz}, \mathrm{CDCl}_{3}\right.$ ) $\delta$ ppm: $7.51(6 \mathrm{H}, \mathrm{m}), 7.30-7.20(9 \mathrm{H}, \mathrm{m}), 4.93(1 \mathrm{H}, \mathrm{t}, J=6.6 \mathrm{~Hz}), 4.77(0.5 \mathrm{H}, \mathrm{dd}, J=6.7,6.7 \mathrm{~Hz}), 4.68$ $(0.5 \mathrm{H}, \mathrm{dd}, J=9.0,5.5 \mathrm{~Hz}), 4.00(1 \mathrm{H}, \mathrm{dd}, J=5.7,5.7 \mathrm{~Hz}), 3.79(0.5 \mathrm{H}, \mathrm{m}), 3.73(0.5 \mathrm{H}, \mathrm{m}), 3.47-3.36$ $(1 \mathrm{H}, \mathrm{m}), 2.31(0.5 \mathrm{H}, \mathrm{m}), 2.12-1.88(3.5 \mathrm{H}, \mathrm{m}), 1.82-1.73(2.5 \mathrm{H}, \mathrm{m}), 1.63(3 \mathrm{H}, \mathrm{m}), 1.54(3 \mathrm{H}, \mathrm{m}), 1.48$ 
(3H, m), 1.57-1.43 (2.5H, m), 1.41-1.31 (2H, m), $1.17(1.5 \mathrm{H}, \mathrm{m}), 1.14(1.5 \mathrm{H}, \mathrm{m}), 1.07(3 \mathrm{H}, \mathrm{m}) ;{ }^{13} \mathrm{C}-$ NMR (100 MHz, $\left.\mathrm{CDCl}_{3}\right) \delta$ ppm: 145.1, 140.4, 140.3, 133.7, 133.2, 129.0, 127.5, 127.5, 126.9, 126.9, $122.1,121.4,120.6,120.4,87.2,87.2,79.5,78.5,77.2,72.6,72.3,69.0,68.9,59.1,47.3,47.2,39.6$, 33.1, 33.0, 30.3, 30.1, 30.1, 29.6, 29.2, 26.1, 25.8, 25.7, 22.4, 22.4, 22.3, 22.3, 22.2, 17.7, 12.7, 11.6; HRESIMS $(\mathrm{m} / \mathrm{z})$ calcd. for $\mathrm{C}_{38} \mathrm{H}_{48} \mathrm{O}_{3} \mathrm{Na}(\mathrm{M}+\mathrm{Na})^{+}$575.3501, found 575.3522; Anal. Calcd. for $\mathrm{C}_{38} \mathrm{H}_{48} \mathrm{O}_{3}$ : C, 82.56; H, 8.75. Found: C, 82.52; H, 8.60.

3.18. (3E,5R)-1-((1S,2R)-2-Acetyl-1-methylcyclopentyl)-4,8-dimethyl-5-trityloxynona-3,7-dien-1-one and (3E,5S)-1-((1S,2R)-2-acetyl-1-methylcyclopentyl)-4,8-dimethyl-5-trityloxynona-3,7-dien-1-one (19)

To a cold $\left(-78{ }^{\circ} \mathrm{C}\right)$ solution of TFAA $(67.6 \mathrm{mg}, 0.322 \mathrm{mmol})$ in $\mathrm{CH}_{2} \mathrm{Cl}_{2}(100 \mu \mathrm{L})$ was added DMSO (33.6 mg, $0.430 \mathrm{mmol})$ in $\mathrm{CH}_{2} \mathrm{Cl}_{2}(100 \mu \mathrm{L})$. The mixture was stirred at $-78{ }^{\circ} \mathrm{C}$ for $30 \mathrm{~min}$, treated with a solution of the diastereomeric mixture of diol 18 (29.6 mg, $0.054 \mathrm{mmol})$ in $\mathrm{CH}_{2} \mathrm{Cl}_{2}$ $(340 \mu \mathrm{L})$, stirred for $2 \mathrm{hr}$ and then $\mathrm{Et}_{3} \mathrm{~N}(54.3 \mathrm{mg}, 0.537 \mathrm{mmol})$ was added. The mixture was warmed to r.t. and stirred for $30 \mathrm{~min}$. The mixture was diluted with $\mathrm{Et}_{2} \mathrm{O}$, washed with saturated aqueous $\mathrm{NaHCO}_{3}$ solution, $\mathrm{H}_{2} \mathrm{O}$ and brine and then dried. Removal of the solvent gave a residue which was then purified by silica gel column chromatography (hexane/AcOEt $=6: 1$ ) to generate a diastereomeric mixture of diketone $19\left(27.9 \mathrm{mg}, 95 \%\right.$ yield) as a colorless oil. IR (neat) $2965,1705 \mathrm{~cm}^{-1}$; ${ }^{1} \mathrm{H}-\mathrm{NMR}$ $\left(400 \mathrm{MHz}, \mathrm{CDCl}_{3}\right) \delta$ ppm: $7.49(6 \mathrm{H}, \mathrm{m}), 7.26-7.16(9 \mathrm{H}, \mathrm{m}), 4.95(1 \mathrm{H}, \mathrm{t}, J=6.1 \mathrm{~Hz}), 4.81(1 \mathrm{H}, \mathrm{t}$, $J=7.3 \mathrm{~Hz}), 3.92(1 \mathrm{H}, \mathrm{dd}, J=8.8,4.7 \mathrm{~Hz}), 2.92(2 \mathrm{H}, \mathrm{m}), 2.78(1 \mathrm{H}, \mathrm{dd}, J=8.6,5.0 \mathrm{~Hz}), 2.23-1.72(6 \mathrm{H}$, m), $2.15(3 \mathrm{H}, \mathrm{s}), 1.63-1.55(2 \mathrm{H}, \mathrm{m}), 1.58(3 \mathrm{H}, \mathrm{s}), 1.48(3 \mathrm{H}, \mathrm{s}), 1.42(3 \mathrm{H}, \mathrm{s}), 1.21(3 \mathrm{H}, \mathrm{s}) ;{ }^{13} \mathrm{C}-\mathrm{NMR}$ $\left(100 \mathrm{MHz}, \mathrm{CDCl}_{3}\right) \delta$ ppm: 212.2, 210.7, 145.2, 137.6, 132.4, 129.2, 127.5, 126.8, 120.5, 118.7, 87.2, 79.3, 77.2, 60.9, 59.8, 37.9, 35.4, 33.5, 29.9, 27.6, 25.8, 25.2, 22.4, 17.8, 12.1; HRESIMS (m/z) calcd. for $\mathrm{C}_{38} \mathrm{H}_{44} \mathrm{O}_{3} \mathrm{Na}(\mathrm{M}+\mathrm{Na})^{+}$571.3188, found 571.3196. Anal. Calcd. for $\mathrm{C}_{38} \mathrm{H}_{44} \mathrm{O}_{3}: \mathrm{C}, 83.17 ; \mathrm{H}, 8.08$. Found: C, 83.12; H, 8.21.

\subsection{Diastereomeric mixture of secoxestenone (20)}

To a solution of the diastereomeric mixture of diketone 19 (76.2 $\mathrm{mg}, 0.139 \mathrm{mmol})$ in $\mathrm{CH}_{2} \mathrm{Cl}_{2}(14$ $\mathrm{mL})$ was added $\mathrm{Yb}(\mathrm{OTf})_{3}(172 \mathrm{mg}, 0.277 \mathrm{mmol})$ at r.t. After stirring for $30 \mathrm{~min}$, the mixture was diluted with $\mathrm{Et}_{2} \mathrm{O}$. To this was added $\mathrm{NaHCO}_{3}$ and the mixture was then filtered through a silica gel pad. Removal of the solvent gave a residue which was then purified by silica gel column chromatography (hexane/AcOEt $=1: 1$ ) to generate a diastereomeric mixture of secoxestenone 20 (33.5 $\mathrm{mg}, 79 \%$ yield) as a colorless oil. IR (neat) 3448, 2965, $1706 \mathrm{~cm}^{-1} ;{ }^{1} \mathrm{H}-\mathrm{NMR}\left(400 \mathrm{MHz}, \mathrm{CDCl}_{3}\right) \delta$ ppm: $5.60(1 \mathrm{H}, \mathrm{m}), 5.10(1 \mathrm{H}, \mathrm{m}), 4.05(1 \mathrm{H}, \mathrm{m}), 3.27(2 \mathrm{H}, \mathrm{m}), 2.85(1 \mathrm{H}, \mathrm{m}), 2.27(3 \mathrm{H}, \mathrm{m}), 2.16(3 \mathrm{H}$, m), $2.09(1 \mathrm{H}, \mathrm{m}), 1.91-1.75(3 \mathrm{H}, \mathrm{m}), 1.71(3 \mathrm{H}, \mathrm{s}), 1.66(1 \mathrm{H}, \mathrm{m}), 1.63(3 \mathrm{H}, \mathrm{s}), 1.63(3 \mathrm{H}, \mathrm{s}), 1.28(3 \mathrm{H}$ $\mathrm{m}) ;{ }^{13} \mathrm{C}-\mathrm{NMR}\left(100 \mathrm{MHz}, \mathrm{CDCl}_{3}\right) \delta$ ppm: 212.8, 210.8, 148.5, 141.9, 134.7, 120.8, 120.1, 118.2, 77.2, 76.9, 61.3, 60.4, 59.7, 37.8, 35.9, 35.5, 34.0, 30.2, 29.8, 27.8, 27.7, 25.9, 25.7, 25.6, 25.3, 22.4, 18.0, 12.3, 12.1; HRESIMS (m/z) calcd. for $\mathrm{C}_{19} \mathrm{H}_{30} \mathrm{O}_{3} \mathrm{Na}(\mathrm{M}+\mathrm{Na})^{+}$329.2093, found 329.2085. Anal. Calcd. for $\mathrm{C}_{19} \mathrm{H}_{30} \mathrm{O}_{3}$ : C, 74.47; H, 9.87. Found: C, 74.29; H, 9.85. 


\subsection{Xestenone (21) and 12-epi-xestenone (12-epi-21)}

To a solution of the diastereomeric mixture of secoxestenone 20 (26.5 mg, $0.087 \mathrm{mmol})$ in $\mathrm{MeOH}$ $(6.7 \mathrm{~mL})$ was added $0.1 \mathrm{M} \mathrm{NaOH}$ aqueous solution $(21.6 \mathrm{~mL})$ at r.t. The mixture was stirred for $30 \mathrm{~min}$, neutralized with $1.0 \mathrm{M} \mathrm{HCl}$ aqueous solution, diluted with $\mathrm{Et}_{2} \mathrm{O}$, washed with $\mathrm{H}_{2} \mathrm{O}$ and brine, dried and then concentrated under reduced pressure. The resultant residue was purified by silica gel column chromatography (hexane/AcOEt $=4: 1)$ to give a mixture of 21 and 12-epi-21 $(22.0 \mathrm{mg}, 88 \%$ yield) as a colorless oil. The above mixture was subjected to HPLC (CHIRALPAK IA, $1.0 \mathrm{~cm} \times 25 \mathrm{~cm}$, hexane $/ \mathrm{EtOH}=95: 5$, flow rate: $1.0 \mathrm{~mL} / \mathrm{min})$ to give xestenone $21\left(t_{\mathrm{R}}=12.0 \mathrm{~min}\right)$ and 12-epi-21 $\left(t_{\mathrm{R}}=15.0 \mathrm{~min}\right) ; 21:[\alpha]_{\mathrm{D}}{ }^{25}+2.2($ c $0.075, \mathrm{MeOH}) ; \mathrm{UV}(\mathrm{sh}, \mathrm{MeOH}) \lambda_{\max } \mathrm{nm}(\varepsilon): 257(6100)$; $\mathrm{CD}(\mathrm{MeOH}) \lambda_{\text {ext }} \mathrm{nm}[\theta]: 323$ (+87,000), 258 (-129,000); IR (neat) 3419, $1685 \mathrm{~cm}^{-1}$; ${ }^{1} \mathrm{H}-\mathrm{NMR}(600$ $\left.\mathrm{MHz}, \mathrm{CDCl}_{3}\right) \delta$ ppm: $5.93(1 \mathrm{H}, \mathrm{s}), 5.18(1 \mathrm{H}$, br t, $J=6.9 \mathrm{~Hz}), 4.18(1 \mathrm{H}, \mathrm{t}, J=6.4 \mathrm{~Hz}), 2.71(1 \mathrm{H}, \mathrm{d}$, $J=9.1 \mathrm{~Hz}), 2.35(2 \mathrm{H}, \mathrm{m}), 1.96(3 \mathrm{H}, \mathrm{s}), 1.93(1 \mathrm{H}, \mathrm{m}), 1.90(1 \mathrm{H}, \mathrm{br} \mathrm{s}), 1.82(1 \mathrm{H}, \mathrm{m}), 1.75(3 \mathrm{H}, \mathrm{s}), 1.69$ $(1 \mathrm{H}, \mathrm{m}), 1.67(3 \mathrm{H}, \mathrm{s}), 1.64(1 \mathrm{H}, \mathrm{m}), 1.55(3 \mathrm{H}, \mathrm{s}), 1.35(1 \mathrm{H}, \mathrm{m}), 1.25(1 \mathrm{H}, \mathrm{m}), 1.21(3 \mathrm{H}, \mathrm{s}) ;{ }^{13} \mathrm{C}-\mathrm{NMR}$ $\left(150 \mathrm{MHz}, \mathrm{CDCl}_{3}\right) \delta$ ppm: 212.7, 172.2, 144.3, 137.4, 134.9, 119.9, 115.6, 76.4, 56.7, 54.8, 37.5, 34.2, 28.9, 25.9, 24.8, 22.5, 18.0, 16.7, 14.4; HRESIMS (m/z) calcd. for $\mathrm{C}_{19} \mathrm{H}_{28} \mathrm{O}_{2} \mathrm{Na}(\mathrm{M}+\mathrm{Na})^{+} 311.1987$, found 311.1981. Anal. Calcd. for $\mathrm{C}_{19} \mathrm{H}_{28} \mathrm{O}_{2}$ : C, 79.12; $\mathrm{H}, 9.78$. Found: C, 78.97; $\mathrm{H}, 9.74$. 12-epi-21: $[\alpha]_{\mathrm{D}}{ }^{25}$-113.7 (c 0.085, MeOH); UV (sh, MeOH) $\lambda_{\max } \mathrm{nm}(\varepsilon): 254(2,100) ; \mathrm{CD}(\mathrm{MeOH}) \lambda_{\text {ext }}$

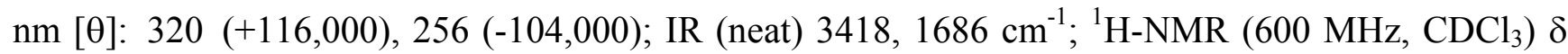
ppm: $5.92(1 \mathrm{H}, \mathrm{s}), 5.16(1 \mathrm{H}, \mathrm{t}, J=7.2 \mathrm{~Hz}), 4.19(1 \mathrm{H}, \mathrm{t}, J=6.3 \mathrm{~Hz}), 2.71(1 \mathrm{H}, \mathrm{d}, J=9.1 \mathrm{~Hz}), 2.35(2 \mathrm{H}$, m), $1.96(3 \mathrm{H}, \mathrm{s}), 1.93(1 \mathrm{H}, \mathrm{m}), 1.81(1 \mathrm{H}, \mathrm{m}), 1.74(3 \mathrm{H}, \mathrm{s}), 1.69(1 \mathrm{H}, \mathrm{m}), 1.67(3 \mathrm{H}, \mathrm{s}), 1.62(1 \mathrm{H}, \mathrm{m})$, $1.55(3 \mathrm{H}, \mathrm{s}), 1.35(1 \mathrm{H}, \mathrm{m}), 1.25(1 \mathrm{H}, \mathrm{m}), 1.22(3 \mathrm{H}, \mathrm{s}) ;{ }^{13} \mathrm{C}-\mathrm{NMR}\left(150 \mathrm{MHz}, \mathrm{CDCl}_{3}\right) \delta \mathrm{ppm}: 212.8$, 172.2, 144.3, 137.4, 134.8, 119.9, 115.9, 76.5, 56.7, 54.7, 37.5, 34.1, 28.9, 25.9, 24.8, 22.5, 18.0, 16.7, 14.1; HRESIMS $(\mathrm{m} / \mathrm{z})$ calcd. for $\mathrm{C}_{19} \mathrm{H}_{28} \mathrm{O}_{2} \mathrm{Na}(\mathrm{M}+\mathrm{Na})^{+}$311.1987, found 311.1975. Anal. Calcd. for $\mathrm{C}_{19} \mathrm{H}_{28} \mathrm{O}_{2}$ : C, 79.12; H, 9.78. Found: C, 79.03; H, 9.88 .

\subsection{General procedure for the synthesis of MPA ester}

To a solution of xestenone (21) in $\mathrm{CH}_{2} \mathrm{Cl}_{2}$ were added DCC, DMAP and (S)-(+)- or (R)-(-)- $\alpha-$ methoxyphenylacetic acid at r.t. After stirring for $30 \mathrm{~min}$ at $40{ }^{\circ} \mathrm{C}$ the mixture was concentrated. Removal of the solvent gave a residue which was then purified by silica gel column chromatography (hexane/AcOEt $=6: 1)$ to generate $(S)$ - or $(R)$-MPA ester. $(S)$-MPA ester: ${ }^{1} \mathrm{H}-\mathrm{NMR}\left(400 \mathrm{MHz}, \mathrm{CDCl}_{3}\right)$ $\delta$ ppm: 7.44-7.27 (5H, m), $5.66(1 \mathrm{H}, \mathrm{s}), 5.26(1 \mathrm{H}, \mathrm{dd}, J=7.9,5.7 \mathrm{~Hz}), 5.06(1 \mathrm{H}, \mathrm{m}), 4.75(1 \mathrm{H}, \mathrm{s}), 3.43$ $(3 \mathrm{H}, \mathrm{s}), 2.62(1 \mathrm{H}, \mathrm{d}, J=9.2 \mathrm{~Hz}), 2.39(2 \mathrm{H}, \mathrm{m}), 1.88(1 \mathrm{H}, \mathrm{m}), 1.76(1 \mathrm{H}, \mathrm{m}), 1.70(3 \mathrm{H}, \mathrm{s}), 1.68(3 \mathrm{H}, \mathrm{s})$, $1.61(3 \mathrm{H}, \mathrm{s}), 1.59(2 \mathrm{H}, \mathrm{m}), 1.30(1 \mathrm{H}, \mathrm{m}), 1.26(3 \mathrm{H}, \mathrm{br} \mathrm{s}), 1.19(1 \mathrm{H}, \mathrm{m}), 1.15(3 \mathrm{H}, \mathrm{s}) ;{ }^{13} \mathrm{C}-\mathrm{NMR}$ $\left(150 \mathrm{MHz}, \mathrm{CDCl}_{3}\right) \delta$ ppm: 211.9, 169.8, 139.2, 137.0, 134.6, 128.8, 128.5, 128.2, 127.2, 119.0, 118.1, 117.8, 82.6, 79.0, 56.6, 54.7, 37.4, 31.8, 29.7, 28.8, 25.8, 24.7, 22.5, 18.0, 16.5, 14.4; HRESIMS (m/z) calcd. for $\mathrm{C}_{28} \mathrm{H}_{36} \mathrm{O}_{4} \mathrm{Na}(\mathrm{M}+\mathrm{Na})^{+}$459.2511, found 459.2521; (R)-MPA ester: ${ }^{1} \mathrm{H}-\mathrm{NMR}(400 \mathrm{MHz}$, $\left.\mathrm{CDCl}_{3}\right) \delta$ ppm: 7.45-7.29 (5H, m), $5.88(1 \mathrm{H}, \mathrm{s}), 5.24(1 \mathrm{H}, \mathrm{dd}, J=7.7,5.7 \mathrm{~Hz}), 4.80(1 \mathrm{H}, \mathrm{m}), 4.77(1 \mathrm{H}$, s), $3.43(3 \mathrm{H}, \mathrm{s}), 2.67(1 \mathrm{H}, \mathrm{d}, J=9.0 \mathrm{~Hz}), 2.28(2 \mathrm{H}, \mathrm{m}), 1.91(1 \mathrm{H}, \mathrm{m}), 1.85(3 \mathrm{H}, \mathrm{s}), 1.81-1.59(3 \mathrm{H}, \mathrm{m})$, $1.53(3 \mathrm{H}, \mathrm{s}), 1.48(3 \mathrm{H}$, br s$), 1.47(3 \mathrm{H}, \mathrm{s}), 1.33(1 \mathrm{H}, \mathrm{m}), 1.22(1 \mathrm{H}, \mathrm{m}), 1.19(3 \mathrm{H}, \mathrm{s}) ;{ }^{13} \mathrm{C}-\mathrm{NMR}$ $\left(150 \mathrm{MHz}, \mathrm{CDCl}_{3}\right) \delta$ ppm: 212.1, 170.0, 139.5, 137.0, 134.4, 128.8, 128.5, 128.2, 127.2, 119.0, 118.7, 
$117.8,82.6,79.0,56.7,54.7,37.5,31.9,29.7,28.9,25.6,24.8,22.5,17.8,16.6,16.5 ;$ HRESIMS $(\mathrm{m} / \mathrm{z})$ calcd. for $\mathrm{C}_{28} \mathrm{H}_{36} \mathrm{O}_{4} \mathrm{Na}(\mathrm{M}+\mathrm{Na})^{+} 459.2511$, found 459.2501 .

\subsection{Conversion of 12-epi-21 to 21}

To a solution of 12-epi-21 $(1.7 \mathrm{mg}, 0.006 \mathrm{mmol})$ in THF $(59 \mu \mathrm{L})$ were added $\mathrm{Ph}_{3} \mathrm{P}(2.3 \mathrm{mg}$, $0.009 \mathrm{mmol})$ and $p-\mathrm{NO}_{2} \mathrm{BzOH}(1.5 \mathrm{mg}, 0.009 \mathrm{mmol})$ at r.t. After stirring for $10 \mathrm{~min}$, DIAD (1.8 mg, $0.009 \mathrm{mmol}$ ) was added and the mixture was stirred for an additional $2 \mathrm{hr}$. The crude mixture was diluted with $\mathrm{Et}_{2} \mathrm{O}$ and filtered through a silica gel pad. The filtrate was then concentrated to afford the crude ester. To a solution of the crude ester in $\mathrm{MeOH}(200 \mu \mathrm{L})$ was added $\mathrm{K}_{2} \mathrm{CO}_{3}(12.2 \mathrm{mg}$, $0.088 \mathrm{mmol}$ ) at r.t. After stirring for $30 \mathrm{~min}$ at the same temperature, the mixture was diluted with $\mathrm{Et}_{2} \mathrm{O}$ and then filtered through a silica gel pad. Removal of the solvent gave a residue which was then purified by silica gel column chromatography (hexane/AcOEt $=2: 1$ ) to generate xestenone (21, $1.6 \mathrm{mg}, 95 \%$ yield).

\section{Conclusions}

The first total synthesis of xestenone has been accomplished via the stereocontrolled one-pot synthesis of cyclopentane derivatives using allyl phenyl sulfone as the key step. Moreover, the authors have determined that the absolute configuration of xestenone is $3 S, 7 S$ and $12 R$.

\section{Acknowledgements}

The authors are grateful to Prof. Raymond J. Andersen of the University of British Columbia for providing the NMR spectra of xestenone.

\section{References and Notes}

1. Andersen, R.J.; Northcote, P.T. Xestenone, a new bicyclic C19 terpenoid from the marine sponge Xestospongia vanilla. Tetrahedron Lett. 1988, 29, 4357-4360.

2. Sipkema, D.; Franssen, M.C.R.; Osinga, R.; Tramper, J.; Wijffels, R.H. Marine sponges as pharmacy. Mar. Biotechnol. 2005, 7, 142-162.

3. Ota, K.; Kurokawa, T.; Kawashima, E.; Miyaoka, H. Stereocontrolled one-pot synthesis of cycloalkane derivatives possessing a quaternary carbon using allyl phenyl sulfone. Tetrahedron 2009, 65, 8668-8676.

4. Andersen, R.J.; Northcote, P.T. Xestolide and secoxestenone, degraded triterpenoids from the sponge Xestospongia vanilla. Can. J. Chem. 1989, 67, 1359-1362.

5. Boeckman, R.K., Jr.; Walters, M.A.; Koyano, H. Regiocontrolled metalation of diethyl $\beta$-dialkylaminovinylphosphonates: A new synthesis of substituted $\beta$-ketophosphonates. Tetrahedron Lett. 1989, 30, 4787-4790.

6. Tago, K.; Arai, M.; Kogen, H. A practical total synthesis of plaunotol via highly Z-selective Wittig olefination of $\alpha$-acetal ketones. J. Chem. Soc., Perkin Trans. I 2000, 2073-2078. 
7. Katsuki, T.; Sharpless, K.B. The first practical method for asymmetric epoxidation. J. Am. Chem. Soc. 1980, 102, 5974-5976.

8. Hartmann, C.; Meyer, V. Iodobenzoic acids. Chem. Ber. 1893, 26, 1727-1732.

9. Luche, J.L. Lanthanides in organic chemistry. 1. Selective 1,2 reductions of conjugated ketones. J. Am. Chem. Soc. 1978, 100, 2226-2227.

10. Trost, B.M.; Belletire, J.L.; Godleski, S.; McDougal, P.G.; Balkovec, J.M. On the use of the Omethylmandelate ester for establishment of absolute configuration of secondary alcohols. J. Org. Chem. 1986, 51, 2370-2374.

11. Mitsunobu, O. The use of diethyl azodicarboxylate and triphenylphosphine in synthesis and transformation of natural products. Synthesis 1981, 1-28.

(C) 2009 by the authors; licensee Molecular Diversity Preservation International, Basel, Switzerland. This article is an open-access article distributed under the terms and conditions of the Creative Commons Attribution license (http://creativecommons.org/licenses/by/3.0/). 